\title{
Constrained Adaptive Backstepping Controller Design for Aircraft Land- ing in Wind Disturbance and Actuator Stuck
}

\author{
Seungho Yoon* and Youdan Kim*** \\ Seoul National University, Seoul, Republic of Korea
}

\section{Sanghyuk Park**}

Korea Aerospace University, Goyang, Republic of Korea

\begin{abstract}
An adaptive backstepping controller is designed for the automatic landing of a fixed-wing aircraft. The backstepping control scheme is adopted by using the nonlinear six degree-of-freedom dynamics of the aircraft during the landing phase. The adaptive law is integrated along with the backstepping controller in order to estimate the aircraft modeling errors as well as the external disturbance. The dynamic constraints of the states and the actuator inputs are taken into account in the parameter adaptation. This is done to prevent an aggressive adaptation and to provide reliable control commands. Numerical simulations were performed to verify the performance of the proposed control law for the landing of the aircraft with the presence of gust and actuator stuck.
\end{abstract}

Key words: Adaptive control, backstepping scheme, hedging technique, command filter, automatic landing

\section{Introduction}

Aircraft landing systems have been continuously developed because the landing phase is the most critical flight stage. According to Boeing's statistical report, $59 \%$ of the commercial aircraft accidents occurred during the approach and landing phases although their flight time comprises only $16 \%$ of the total flight phase [1]. Moreover, $45 \%$ of the accidents were due to wind turbulences. Therefore, the landing controller should be robust enough for various disturbances such as wind turbulence, unpredictable gust near the ground, and control surface failures. In the conventional instrument landing system, the landing of the fixed-wing aircraft is divided into three phases: a glide phase, a flare phase, and a touchdown phase [2]. The aircraft approaches to the runway while tracking a specified glide slope in the glide phase and it follows an exponential path to slow the descent rate in the flare phase. VHF Omni Range (VOR) and radio beam are necessary to guide the aircraft to the pre-designed glide slope and runway. This system requires additional instruments in the aircraft to measure the signals that are transmitted from the ground system. Studies on the automatic landing controller have been performed recently by applying various control techniques: Proportional-Integral-Derivative (PID) control, Linear Quadratic Regulator (LQR), $\mathrm{H}_{2} / \mathrm{H}_{\infty}$ control, feedback linearization, backstepping control, sliding mode control, adaptive control, neural network, fuzzy logic, etc.

The classical approaches of the landing controller use the PID control and the LQR for a linear model of the aircraft [2]. In order to deal with the uncertainties in the linear model, robust control techniques such as $H_{2} / H_{\infty}$ control theory and quantitative feedback theory are applied [3-6]. Niewoehner and Kaminer designed a carrier landing controller of the F-14 aircraft by translating typical Single-Input Single-Output (SISO) design specification into the $H_{\infty}$ synthesis problem [3]. Fialho et al. constructed a linear fractional model which
This is an Open Access article distributed under the terms of the Creative Commons Attribution Non-Commercial License (http://creativecommons.org/licenses/by$\mathrm{nc} / 3.0 /$ ) which permits unrestricted non-commercial use, distribution and reproduction in any medium, provided the original work is properly cited. (c) * Postdoctoral Research Associate

** Assistant Professor

*** Professor, Corresponding author: E-mail: ydkim@snu.ac.kr 
is based on multiple linear models and designed a gainscheduled controller that provides a uniform handling quality over angle-of-attack variations [4]. Liao et al. developed a fault-tolerant landing controller based on the $\mathrm{H}_{2}$ technique by using multiple fault models [5]. Wagner and Valasek designed an aircraft landing controller which provides allaround robustness by comparing the quantitative feedback theory and the proportional-integral controller [6]. However, the PID control, LQR, and robust controllers are mostly dependent on the pre-determined margins and they bounded uncertainties of the linear models.

The alternative approach to the model-based linear controller is a nonlinear adaptive control. In order to deal with the nonlinear aircraft model, feedback linearization and backstepping technique are considered $[7,8]$. Biju et al. designed a landing controller by using the feedback linearization [9]. Ju et al. developed a glide-slope tracking controller by using a non-adaptive backstepping technique [10]. However, in the nonlinear model highly nonlinear modeling errors and unknown parameter errors still exist. In order to deal with the uncertainties, various adaptive control theories are introduced: neural network, parameter adaptation, fuzzy logic, and genetic algorithm [11-16]. Saini et al. compared adaptive critic based neural networks with the PID controller in the aircraft landing [11]. Naikal et al. combined the classical landing controller with the radial basis function neural network [12]. Yoon et al. designed the backstepping controller to land the fixed-wing aircraft by using parameter adaptation [13]. Lee et al. compared the adaptive sliding mode controller with the feedback linearization in the landing of a quad-rotor [14]. Nho and Agarwal developed a fuzzy logic control system for the automatic landing of both linear and nonlinear aircraft models [15]. Ha modified the fuzzy logic controller through the optimization of the gain-scheduled controller by using a genetic algorithm in the automatic landing and touchdown flight [16].

In order to design the adaptive landing controller, wind disturbance and actuator failure near the ground should be considered. The wind disturbance increases the glideslope tracking error and it may cause a severe accident during the landing phase. In Ref. 12, the deterministic wind, wind turbulence, and wind-shear model are considered for the design of the landing controller. In Ref. 16, wind shear turbulence near the ground is modeled as head wind, crosswind, and downdraft, and the influence of the strong wind on the aircraft is analyzed. On the other hand, the probability of the actuator fault during the landing phase is higher compared to that during the cruise phase. This is due to the control surfaces of the aircraft which are commanded more rapidly and frequently during the landing phase. The actuator failure of the control surfaces during the landing phase causes more serious problem than during the cruise phase. These problems arise due to limited time and space for recovery. In Ref. 12, the actuator fault of multiple control surfaces is taken into account in the design of adaptive backstepping neural controller.

In this study, the automatic landing controller of the fixedwing aircraft is designed by using the backstepping scheme and the constrained adaptation technique. Nonlinear six degree-of-freedom aircraft model is considered for the design of the backstepping controller that tracks a desired glide slope toward the runway. In order to estimate the modeling errors of aerodynamic coefficients in the nonlinear model, the adaptive parameter estimation of the nonlinear function is adopted. The landing controller proposed in this study is different from the previous landing controllers in that the aggressive adaptation due to the wind disturbance and actuator failure is prevented by applying the hedging techniques. Even though the stability of the adaptive backstepping scheme is strictly addressed in the controller design, the dynamic characteristics of the system may cause an undesired behavior in the adaptive control. Moreover, each control surface of the aircraft has constraints which include magnitude saturation and rate limit. Therefore, the dynamic characteristics and the input constraints of the aircraft should be considered in the design of the adaptive control. In Ref. 17, a pseudo control hedging technique is proposed for the design of neural networks, and in Ref. 18, the pseudo control hedging is combined along with the training signal hedging techniques in the design of adaptive backstepping controller. In this study, the pseudo control hedging technique and the training signal hedging technique are combined with the command filter to design a reliable adaptive landing controller. In order to verify the landing performance of the constrained adaptive backstepping scheme, the glide-slope tracking errors of the constrained adaptive controller is compared with those of an unconstrained adaptive controller, a non-adaptive backstepping controller, and a gain-scheduled proportionalintegral controller. In numerical simulation, wind gust and actuator stuck during the landing phase are included for the evaluation of the landing performance.

The rest of this paper is organized as follows: Section 2 explains the landing procedure and the nonlinear dynamics of the fixed-wing aircraft. Section 3 describes the design of the adaptive backstepping controller by using the unconstrained and constrained adaptation schemes. In Section 4, nonlinear simulation result and analysis are presented. Conclusions are given in Section 5. In Appendix, the design procedure of the 
constrained adaptive backstepping controller is explained in detail.

\section{Aircraft Landing Model}

The automatic landing of the fixed-wing aircraft is divided into three phases: final approach, flare, and touchdown [19]. Figure 1 shows the conventional landing configuration of the fixed-wing aircraft. As shown in Fig. $1,\left(p_{x}, p_{y}, p_{z}\right)$ is the current position of the aircraft. In the final approach phase, if no crosswind is considered, the longitudinal axis of the aircraft should be aligned along with the centerline of the runway and the pitch angle of the aircraft is adjusted to track the desired glide slope. In the flare phase, the altitude of the aircraft is smoothly and slowly changed into the runway altitude. Finally in the touchdown phase, the aircraft touches down on the ground. The aircraft is controlled to have a positive angle of attack when the main wheels touch the ground first, and then the nose wheel descends gradually while the aircraft decelerates. If the crosswind is accounted in the final approach phase and in the flare phase then, the ground track of the aircraft is aligned with the centerline of the runway by the crab method or the wing-low method [19]. The crab angle or the roll angle maintained throughout the final approach phase should be removed right before touchdown in order to align the longitudinal axis of the aircraft with the centerline of the runway.

In this study, the six degree-of-freedom nonlinear dynamics of the fixed-wing aircraft is considered [2]. The force equations in the stability axis are represented as follows,

$$
\begin{aligned}
\dot{V}_{T}=g_{X}+\frac{\cos \alpha \cos \beta}{m}\left(X_{A}+\right. & \left.X_{T}\right)+\frac{\sin \beta}{m}\left(Y_{A}+Y_{T}\right) \\
& +\frac{\sin \alpha \cos \beta}{m}\left(Z_{A}+Z_{T}\right)
\end{aligned}
$$

$$
\begin{aligned}
\dot{\alpha}=- & p \cos \alpha \tan \beta+q-r \sin \alpha \tan \beta+g_{Z} \\
& -\frac{\sin \alpha}{m V_{T} \cos \beta}\left(X_{A}+X_{T}\right)+\frac{\cos \alpha}{m V_{T} \cos \beta}\left(Z_{A}+Z_{T}\right)
\end{aligned}
$$

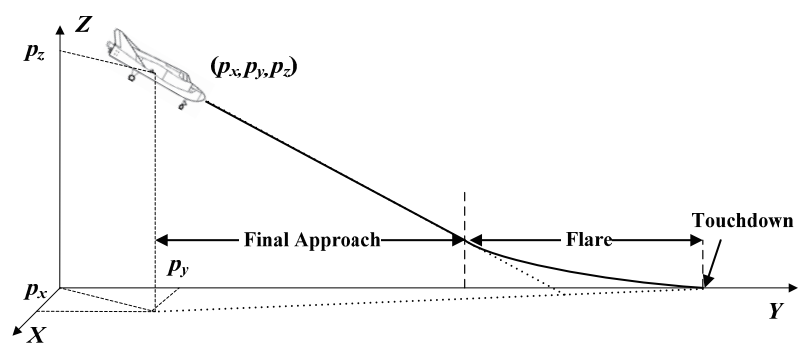

Fig. 1. Landing configuration of a fixed-wing aircraft

$$
\begin{aligned}
\dot{\beta}=p \sin \alpha- & r \cos \alpha+g_{Y}-\frac{\cos \alpha \sin \beta}{m V_{T}}\left(X_{A}+X_{T}\right) \\
+ & \frac{\cos \beta}{m V_{T}}\left(Y_{A}+Y_{T}\right)-\frac{\sin \alpha \sin \beta}{m V_{T}}\left(Z_{A}+Z_{T}\right)
\end{aligned}
$$

where, $V_{T}$ is the true velocity, $\alpha$ is the angle of attack, $\beta$ is the sideslip angle, $p, q$, and $r$ are angular rates in the $X$, $Y$, and $Z$ directions, respectively. Moreover, $m$ is the mass of the aircraft, $g_{X}, g_{Y}$, and $g_{Z}$ are gravitational acceleration components in the stability axis, $X_{A}, Y_{A}$, and $Z_{A}$. They are aerodynamic forces in the $X, Y$, and $Z$ directions. $X_{T}, Y_{T}$, and $Z_{T}$ are the thrust forces in the $X, Y$, and $Z$ directions, respectively.

The moment equations are represented as follows,

$$
\begin{gathered}
\dot{p}=I_{1} p q+I_{2} q r+I_{3} L+I_{4} N \\
\dot{q}=I_{5} p r-I_{6}\left(p^{2}-r^{2}\right)+I_{7} M \\
\dot{r}=I_{8} p q-I_{1} q r+I_{4} L+I_{9} N
\end{gathered}
$$

where, $L, M$, and $N$ are the angular moments in the $X, Y$, and $Z$ axes, respectively, and $I_{i}$ are defined as follows [20]:

$$
\begin{aligned}
& I_{1}=\frac{I_{x z}\left(I_{x}-I_{y}+I_{z}\right)}{I_{x} I_{z}-I_{x z}{ }^{2}}, I_{2}=-\frac{I_{z}\left(I_{z}-I_{y}\right)+I_{x z}{ }^{2}}{I_{x} I_{z}-I_{x z}{ }^{2}}, \\
& I_{3}=\frac{I_{z}}{I_{x} I_{z}-I_{x z}{ }^{2}}, I_{4}=\frac{I_{x z}}{I_{x} I_{z}-I_{x z}{ }^{2}}, I_{5}=\frac{I_{z}-I_{x}}{I_{y}}, I_{6}=\frac{I_{x z}}{I_{y}}(7) \\
& I_{7}=\frac{1}{I_{y}}, I_{8}=\frac{I_{x}\left(I_{x}-I_{y}\right)+I_{x z}{ }^{2}}{I_{x} I_{z}-I_{x z}{ }^{2}}, I_{9}=\frac{I_{x}}{I_{x} I_{z}-I_{x z}{ }^{2}}
\end{aligned}
$$

where, $I_{x}, I_{y}$, and $I_{z}$ are the moments of inertia about $x$-axis, $y$-axis, and $z$-axis, respectively, and $I_{x z}$ is the cross-product of inertia about $x$-axis and $z$-axis. The kinematic equations are represented as follows:

$$
\begin{aligned}
& \dot{\phi}=p+(q \sin \phi+r \cos \phi) \tan \theta \\
& \dot{\theta}=q \cos \phi-r \sin \phi \\
& \dot{\psi}=(q \sin \phi+r \cos \phi) / \cos \theta
\end{aligned}
$$

Let us define the states $x_{1} \in R^{3}, x_{2} \in R^{3}$, and the control input $u \in R^{3}$ as,

$$
\begin{aligned}
& x_{1}=\left[\begin{array}{lll}
\phi & \alpha & \beta
\end{array}\right]^{T} \\
& x_{2}=\left[\begin{array}{lll}
p & q & r
\end{array}\right]^{T} \\
& u=\left[\begin{array}{lll}
\delta a & \delta e & \delta r
\end{array}\right]^{T}
\end{aligned}
$$


where, $\delta_{a}$ is an aileron control input, $\delta_{e}$ is an elevator control input, and $\delta_{r}$ is a rudder control input. By using the state and control input vectors which are defined in Eqs. (11)-(13), Eqs. (2)-(8) can be rewritten as,

$$
\begin{aligned}
& \dot{x}_{1}=f_{1}+g_{1} x_{2}+h_{1} u \\
& \dot{x}_{2}=f_{2}+g_{2} u
\end{aligned}
$$

where, $f_{1}, f_{2}, g_{1}, g_{2}$ and $h_{1}$ are defined as,

$$
\begin{array}{r}
f_{\mathrm{l}}=\frac{\bar{q} S}{m V_{T}}\left[\begin{array}{c}
0 \\
-C_{X} \frac{\sin \alpha}{\cos \beta}+C_{Z} \frac{\cos \alpha}{\cos \beta} \\
-C_{X} \cos \alpha \sin \beta+C_{Y} \cos \beta-C_{Z} \sin \alpha \sin \beta
\end{array}\right]+\frac{F_{T}}{m V_{T}}\left[\begin{array}{c}
0 \\
-\frac{\sin \alpha}{\cos \beta} \\
0 \\
-\cos \alpha \sin \beta
\end{array}\right] \\
+\frac{g}{V_{T}}\left[\begin{array}{c}
\sin \alpha \sin \theta+\cos \alpha \cos \phi \cos \theta \\
\cos \alpha \sin \beta \sin \theta+\cos \beta \sin \phi \cos \theta-\sin \alpha \sin \beta \cos \phi \cos \theta
\end{array}\right]
\end{array}
$$

$$
\begin{aligned}
f_{2} & =\left[\begin{array}{c}
I_{1} p q+I_{2} q r \\
I_{5} p r-I_{6}\left(p^{2}-r^{2}\right) \\
I_{8} p q-I_{1} q r
\end{array}\right]+\bar{q} S\left[\begin{array}{c}
I_{3} b C_{l}+I_{4} b C_{n} \\
I_{7} \bar{c} C_{m 0} \\
I_{4} b C_{l}+I_{9} b C_{n}
\end{array}\right] \\
& +\frac{\bar{q} S}{2 V_{T}}\left[\begin{array}{ccc}
I_{3} b^{2} C_{l p}+I_{4} b^{2} C_{n p} & 0 & I_{3} b^{2} C_{l r}+I_{4} b^{2} C_{n r} \\
0 & I_{7} \bar{c}^{2} C_{m q} & 0 \\
I_{4} b^{2} C_{l p}+I_{9} b^{2} C_{n p} & 0 & I_{4} b^{2} C_{l p}+I_{9} b^{2} C_{n p}
\end{array}\right]\left[\begin{array}{l}
p \\
q \\
r
\end{array}\right]
\end{aligned}
$$

$$
g_{1}=\left[\begin{array}{ccc}
1 & \sin \phi \tan \theta & \cos \phi \tan \theta \\
-\cos \alpha \tan \beta & 1 & \sin \alpha \tan \beta \\
\sin \alpha & 0 & -\cos \alpha
\end{array}\right]
$$

$$
g_{2}=\bar{q} S\left[\begin{array}{ccc}
I_{3} b C_{l \delta a}+I_{4} b C_{n \delta a} & 0 & I_{3} b C_{l \delta r}+I_{4} b C_{n \delta r} \\
0 & I_{7} \bar{c} C_{m \delta e} & 0 \\
I_{4} b C_{l \delta a}+I_{9} b C_{n \delta a} & 0 & I_{4} b C_{l \delta r}+I_{9} b C_{n \delta r}
\end{array}\right]
$$

$$
\left.h_{1}=\frac{\bar{q} S}{m V_{T}} \mid \begin{array}{ccc}
0 & 0 & 0 \\
-C_{X \delta a} \frac{\sin \alpha}{\cos \beta} & -C_{X \delta \varepsilon} \frac{\sin \alpha}{\cos \beta}+C_{Z \delta \epsilon} \frac{\cos \alpha}{\cos \beta} & 0 \\
-C_{X \delta c} \cos \alpha \sin \beta+C_{Y \delta \alpha} \cos \beta & -C_{X \delta \varepsilon} \cos \alpha \sin \beta-C_{Z \delta \epsilon} \sin \alpha \sin \beta & C_{Y \delta r} \cos \beta
\end{array}\right]
$$

where, $\bar{q}$ is a dynamic pressure, $S$ is a reference area, $b$ is a length of wing span, $\bar{c}$ is a mean aerodynamic chord length, $C_{X}$ is an axial-force coefficient, $C_{Y}$ is a side-force coefficient, $C_{Z}$ is a normal-force coefficient, $C_{l}$ is a rolling-moment coefficient, $C_{m}$ is a pitching-moment coefficient, $C_{n}$ is a yawing-moment coefficient and $F_{T}$ is a thrust force, respectively. The objective of this study is to design the control input, $u$, to guide the aircraft to the runway by tracking the glide-slope command, $x_{1}^{d}$. The glide-slope command is defined along with the glide slope in Section 4.

\section{Adaptive Backstepping Controller Design}

\subsection{Unconstrained Adaptive Backstepping Controller}

In the design of the unconstrained adaptive controller, the dynamic characteristics and the input constraints of the aircraft are not considered. Figure 2 illustrates the schematic diagram of the unconstrained adaptive backstepping controller from the reference command $x_{1}^{d}$ to the control input $u$. As shown in Fig. 2, $u$ is designed to make $x_{1}$ track $x_{1}^{d}$ by using the backstepping scheme. Equations (16) and (17) in the real flight include the aerodynamic uncertainties and the disturbance. As a result the nonlinear functions $f_{1}$ and $f_{2}$ are estimated. The estimation errors are defined as follows,

$$
\begin{gathered}
\tilde{f}_{1}=\hat{f}_{1}-f_{1} \\
\tilde{f}_{2}=\hat{f}_{2}-f_{2}
\end{gathered}
$$

where, $\tilde{f}_{1}$ and $\tilde{f}_{2}$ are the estimation errors, and $\hat{f}_{1}$ and $\hat{f}_{2}$ are the estimates of $f_{1}$ and $f_{2}$. The error between $x_{1}$ and $x_{1}^{d}$, which lies in a convex and compact region $D$ for $t>0$, is defined as follows,

$$
z_{1}=x_{1}-x_{1}^{d}
$$

Consider the following Lyapunov function candidate

$$
V_{1}=\frac{1}{2} z_{1}^{T} z_{1}+\frac{1}{2 c_{1}} \tilde{f}_{1}^{T} \tilde{f}_{1}
$$

where, $c_{1}$ is a positive constant. The time derivative of Eq. (24) is semi-negative definite, if $x_{2}$ and the corresponding adaptation law are chosen as

$$
\begin{aligned}
& x_{2}=g_{1}{ }^{-1}\left[-\hat{f}_{1}-h_{1} u+\dot{x}_{1}^{d}-K_{1} z_{1}\right] \\
& \dot{\tilde{f}}_{1}=\dot{\hat{f}}_{1}-\dot{f}_{1}^{*}=\dot{\hat{f}}_{1}=c_{1} z_{1}
\end{aligned}
$$

where, $K_{1}$ is a positive definite gain matrix and $f_{1}^{*}$ is the nominal function of $f_{1}$. It is to be noted that the right-hand side of Eq. (25) includes the actual control input, $u$. As shown in Eq. (20), during the landing phase, $h_{1}$ is close to the zero

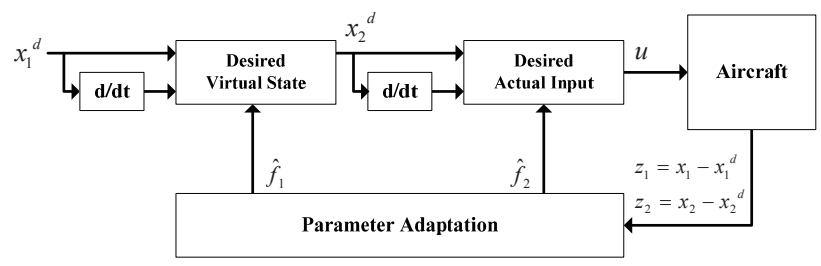

Fig. 2. Unconstrained adaptive backstepping control scheme 
matrix. Therefore, $h_{1} u$ in Eq. (25) can be approximated to a zero vector and ignored in the design of the backstepping controller [20]. Moreover, in the adaptive controller, this term can be treated as an additional uncertainty in $f_{1}$, and it can be compensated by using the adaptation law of Eq. (26).

By substituting Eqs. (14), (21) and (23) into the time derivative of Eq. (24) yields,

$$
\begin{aligned}
\dot{V}_{1} & =z_{1}^{T} \dot{z}_{1}+\frac{1}{c_{1}} \tilde{f}_{1}^{T} \dot{\tilde{f}}_{1} \\
& =z_{1}^{T}\left[\dot{x}_{1}-\dot{x}_{1}^{d}\right]+\frac{1}{c_{1}} \tilde{f}_{1}^{T} \dot{\tilde{f}}_{1} \\
& =z_{1}^{T}\left[f_{1}+g_{1} x_{2}+h_{1} u+\left(\hat{f}_{1}-\hat{f}_{1}\right)-\dot{x}_{1}^{d}\right]+\frac{1}{c_{1}} \tilde{f}_{1}^{T} \dot{\tilde{f}}_{1} \\
& =z_{1}^{T}\left[\hat{f}_{1}+g_{1} x_{2}+h_{1} u-\dot{x}_{1}^{d}\right]-z_{1}^{T} \tilde{f}_{1}+\frac{1}{c_{1}} \tilde{f}_{1}^{T} \dot{\tilde{f}}_{1}
\end{aligned}
$$

Applying Eqs. (25) and (26) to Eq. (27) yields

$$
\dot{V}_{1}=-z_{1}^{T} K_{1} z_{1} \leq 0 \text {. }
$$

Therefore, the state error defined in Eq. (23) can be regulated, and $x_{1}$ converges to $x_{1}^{d}$. In the backstepping scheme, $x_{2}$ in Eq. (25) is considered as the desired state $x_{2}^{d}$ of the real state $x_{2}$. Then, the error between $x_{2}$ and $x_{2}^{d}$ is defined as follows,

$$
z_{2}=x_{2}-x_{2}^{d} .
$$

Consider the following Lyapunov function candidate

$$
V_{2}=\frac{1}{2} z_{1}{ }^{T} z_{1}+\frac{1}{2} z_{2}{ }^{T} z_{2}+\frac{1}{2 c_{1}} \tilde{f}_{1}^{T} \tilde{f}_{1}+\frac{1}{2 c_{2}} \tilde{f}_{2}^{T} \tilde{f}_{2}
$$

where, $c_{2}$ is a positive constant. The time derivative of Eq. (30) is semi-negative definite, if $u$ and the corresponding adaptation law are chosen as

$$
\begin{aligned}
& u=g_{2}{ }^{-1}\left[-\hat{f}_{2}+\dot{x}_{2}^{d}-g_{1}{ }^{T} z_{1}-K_{2} z_{2}\right] \\
& \dot{\tilde{f}}_{2}=\dot{\hat{f}}_{2}-\dot{f}_{2}^{*}=\dot{\hat{f}}_{2}=c_{2} z_{2}
\end{aligned}
$$

where, $K_{2}$ is a positive definite gain matrix and $f_{2}^{*}$ is the nominal function of $f_{2}$. By substituting Eqs. (14), (15), (21)(23), (25), and (29) into the time derivative of Eq. (30) yields

$$
\begin{aligned}
\dot{V}_{2} & =z_{1}^{T} \dot{z}_{1}+z_{2}^{T} \dot{z}_{2}+\frac{1}{c_{1}} \tilde{f}_{1}^{T} \dot{\tilde{f}}_{2}+\frac{1}{c_{2}} \tilde{f}_{2}^{T} \dot{\tilde{f}}_{2} \\
& =z_{1}^{T}\left[f_{1}+g_{1} x_{2}+h_{1} u-\dot{x}_{1}^{d}\right]+z_{2}^{T}\left[f_{2}+g_{2} u-\dot{x}_{2}^{d}\right]+\frac{1}{c_{1}} \tilde{f}_{1}^{T} \dot{\tilde{f}}_{2}+\frac{1}{c_{2}} \tilde{f}_{2}^{T} \dot{\tilde{f}}_{2} \\
& =z_{1}^{T}\left[f_{1}+g_{1} z_{2}+g_{1} x_{2}^{d}+h_{1} u+\left(\hat{f}_{1}-\hat{f}_{1}\right)-\dot{x}_{1}^{d}\right]+z_{2}^{T}\left[f_{2}+g_{2} u+\left(\hat{f}_{2}-\hat{f}_{2}\right)-\dot{x}_{2}^{d}\right]+\frac{1}{c_{1}} \tilde{f}_{1}^{T} \dot{\tilde{f}}_{2}+\frac{1}{c_{2}} \tilde{f}_{2}^{T} \dot{\tilde{f}}_{2} \\
& =z_{1}^{T}\left[g_{1} z_{2}-K_{1} z_{1}\right]+z_{2}^{T}\left[\hat{f}_{2}+g_{2} u-\dot{x}_{2}^{d}\right]-z_{1}^{T} \tilde{f}_{1}-z_{2}^{T} \tilde{f}_{2}+\frac{1}{c_{1}} \tilde{f}_{1}^{T} \dot{\tilde{f}}_{2}+\frac{1}{c_{2}} \tilde{f}_{2}^{T} \dot{\tilde{f}}_{2}
\end{aligned}
$$

Finally, by applying Eqs. (26), (31), and (32) to Eq. (33) yields

$$
\dot{V}_{2}=-z_{1}^{T} K_{1} z_{1}-z_{2}^{T} K_{2} z_{2} \leq 0 .
$$

In summary, the state errors defined in Eqs. (23) and (29) can be regulated. Therefore, $x_{1}$ converges to $x_{1}^{d}$, and $x_{2}$ converges to $x_{2}^{d}$, respectively. As shown in Fig. 2, the desired control input $u$ in Eq. (31) makes the state $x_{2}$ converge to the desired state $x_{2}^{d}$ in Eq. (25). Similarly, the desired state $x_{2}^{d}$ makes the state $x_{1}$ converge to the reference command $x_{1}^{d}$. The desired intermediate state $x_{2}^{d}$ is called as a virtual input or virtual state in the backstepping scheme.

\subsection{Constrained Adaptive Backstepping Controller}

In the design of the constrained adaptive controller, the dynamic characteristics and the input constraints of the aircraft are considered. The desired state of Eq. (25) and the control input of Eq. (31) do not assure dynamic characteristics and constraints of the aircraft. In the adaptive controller, the desired states and/or control inputmay exceed the magnitude or the rate limitation if the dynamic characteristics of the states and the control input are not considered throughout the design procedure of the adaptation laws. These immoderate states or saturated control input may cause an aggressive adaptation in the adaptive laws of Eqs. (26) and (32). This can make the aircraft unstable or fly beyond the flight envelope. The command filter and the hedging techniques can deal with the dynamic characteristics and constraints such as response time, magnitude saturation, and rate limit [2124]. In Refs. 21 and 22, the adaptive controller is designed in the presence of the input saturation and constraints. Polycarpu et al. approximated the input saturation with the learning technique in the backstepping controller design [23]. Sonneveldt et al. designed the fault-tolerant flight controller by using the constrained adaptive backstepping scheme and neural networks [24]. Table 1 shows the dynamic characteristics of the actuator in the NASA HL-20 aircraft and it is considered in this study [25]. Table 2 shows the dynamic characteristics of the angular state in a general aircraft.

In this study, two techniques are applied to take the aggressive adaptation into account in the landing controller:

Table 1. Dynamic characteristics of control surface actuators (NASA HL-20)

\begin{tabular}{|c|c|c|c|}
\hline $\begin{array}{c}\text { Control } \\
\text { Surface }\end{array}$ & $\begin{array}{c}\text { Min/Max Magnitude } \\
(\mathrm{deg})\end{array}$ & $\begin{array}{c}\text { Frequency } \\
\text { (rad/sec) }\end{array}$ & $\begin{array}{c}\text { Min/Max Rate } \\
\text { (deg/sec) }\end{array}$ \\
\hline Aileron & $-30 \sim 30$ & 44 & $-80 \sim 80$ \\
\hline Elevator & $-60 \sim 60$ & 44 & $-60 \sim 60$ \\
\hline Rudder & $-60 \sim 60$ & 44 & $-120 \sim 120$ \\
\hline
\end{tabular}


the command filter and the signal hedging. First, the command filter provides a filtered signal and its time derivative according to the desired dynamic characteristics. The command filter adopted in this study is a second-order nonlinear filter and it is defined as [18]

$$
\left[\begin{array}{l}
\dot{y}_{1} \\
\dot{y}_{2}
\end{array}\right]=\left[2 \zeta \omega_{n}\left\{s a t_{\text {rate }}\left(\frac{\omega_{n}{ }^{2}}{2 \zeta \omega_{n}}\left[s a t_{\text {mag }}\left(x_{i}^{d}\right)-y_{1}\right]\right)-y_{2}\right\}\right]
$$

where, $y_{1}$ is the filtered output of the original signal $x_{\mathrm{i}}^{d}, y_{2}$ is its time derivative, $\omega_{n}$ and $\zeta$ are the natural frequency and damping ratio of the desired state, and $s a t_{\text {mag }}(x)$ and $s_{\text {rate }}(x)$ are saturation functions that correspond to the magnitude limitation and the rate limitation, respectively. The reference command $x_{1}^{d}$ is filtered into $x_{1}^{f}$, the desired virtual state $x_{2}^{d}$ defined in Eq. (25) is filtered into $x_{2}^{f}$, and the control input $u^{d}$ defined in Eq. (31) is filtered into $u^{f}$. Consequently, the dynamic characteristics of the desired state and the control input are considered in the filtered output, and the time derivatives that are required in Eqs. (25) and (31) are provided by the nonlinear filter. The adaptation errors in Eqs. (23) and (29) are re-defined using the following filtered states.

$$
\begin{aligned}
& z_{1}=x_{1}-x_{1}^{f} \\
& z_{2}=x_{2}-x_{2}^{f}
\end{aligned}
$$

where, $x_{1}^{f}$ is the filtered state of $x_{1}^{d}$ and $x_{2}^{f}$ is the filtered state of $x_{2}^{d}$.

Second, compensating variables are introduced and the training signal and pseudo control hedging techniques are applied to prevent the estimation errors from aggressive adaptation. In the training signal hedging, the adaptation errors defined in Eqs. (36) and (37) are modified as follows [23].

$$
\begin{aligned}
& \bar{z}_{1}=z_{1}-\xi_{1}=\left(x_{1}-x_{1}^{f}\right)-\xi_{1} \\
& \bar{z}_{2}=z_{2}-\xi_{2}=\left(x_{2}-x_{2}^{f}\right)-\xi_{2}
\end{aligned}
$$

where, $\bar{z}_{i}$ is the modified adaptation error, $z_{i}$ is the original adaptation error, and $\xi_{i}$ is the compensating variable. When

Table 2. Dynamic characteristics of the aircraft angular rates

\begin{tabular}{|c|c|c|c|}
\hline $\begin{array}{c}\text { Control } \\
\text { Surface }\end{array}$ & $\begin{array}{c}\text { Min/Max Magnitude } \\
\text { (deg/sec) }\end{array}$ & $\begin{array}{c}\text { Frequency } \\
\text { (rad/sec) }\end{array}$ & Min/Max Rate \\
\hline Roll Rate & $-120 \sim 120$ & 20 & - \\
\hline $\begin{array}{c}\text { Pitch } \\
\text { Rate }\end{array}$ & $-30 \sim 30$ & 20 & - \\
\hline $\begin{array}{c}\text { Yaw } \\
\text { Rate }\end{array}$ & $-15 \sim 15$ & 20 & - \\
\hline
\end{tabular}

the desired state exceeds the filtered state, $\xi_{i}$ reduces $\bar{z}_{i}$ until $x_{2}^{d}$ and $u^{d}$ have feasible values. In the pseudo control hedging, $x_{2}^{d}$ is modified into $x_{2}^{\text {mod }}$ in order to compensate the error between the desired $u^{d}$ defined in Eq. (31) and the filtered $u^{f}$ when $u^{d}$ exceeds $u^{f}$. Consequently, $x_{2}^{\text {mod }}$ and $\xi_{2}$ are defined as follows,

$$
\begin{aligned}
& x_{2}^{\mathrm{mod}}=x_{2}^{d}-\xi_{2} \\
& \dot{\xi}_{2}=-K_{2} \xi_{2}+g_{2}\left(u^{f}-u^{d}\right)
\end{aligned}
$$

In the same way, when $x_{2}^{\text {mod }}$ exceeds $x_{2}^{f}, \xi_{1}$ is activated as follows,

$$
\dot{\xi}_{1}=-K_{1} \xi_{1}+g_{1}\left(x_{2}^{f}-x_{2}^{\mathrm{mod}}\right)
$$

Finally, the desired virtual state in Eq. (25) and the desired control input in Eq. (31) are rewritten using $x_{i}^{f}$ and $\bar{z}_{1}$ as follows,

$$
\begin{aligned}
& x_{2}^{d}=g_{1}^{-1}\left[-\hat{f}_{1}-h_{1} u^{f}+\dot{x}_{1}^{f}-K_{1} z_{1}\right] \\
& u^{d}=g_{2}{ }^{-1}\left[-\hat{f}_{2}+\dot{x}_{2}^{f}-g_{1}^{T} \bar{z}_{1}-K_{2} z_{2}\right]
\end{aligned}
$$

It is to be noted that $\dot{x}_{1}^{f}$ is not the numerical derivative of $x_{1}^{f}$ but the output of the command filter described in Eq. (35). The adaptation laws in Eqs. (26) and (32) are rewritten using $\bar{z}_{i}$ as follows,

$$
\begin{aligned}
& \dot{\tilde{f}}_{1}=\dot{\hat{f}}_{1}=c_{1} \bar{z}_{1} \\
& \dot{\tilde{f}}_{2}=\dot{\hat{f}}_{2}=c_{2} \bar{z}_{2}
\end{aligned}
$$

Now, consider the following Lyapunov function candidate

$$
V_{3}=\frac{1}{2} \bar{z}_{1}^{T} \bar{z}_{1}+\frac{1}{2} \bar{z}_{2}^{T} \bar{z}_{2}+\frac{1}{2 c_{1}} \tilde{f}_{1}^{T} \tilde{f}_{1}+\frac{1}{2 c_{2}} \tilde{f}_{2}^{T} \tilde{f}_{2}
$$

By substituting Eqs. (38)-(40) with $u=u^{f}$ into the time derivative of Eq. (47) yields

$$
\begin{aligned}
\dot{V}_{3}= & \bar{z}_{1}^{T} \dot{\bar{z}}_{1}+\bar{z}_{2}^{T} \dot{z}_{2}+\frac{1}{c_{1}} \tilde{f}_{1}^{T} \dot{\tilde{f}}_{1}+\frac{1}{c_{2}} \tilde{f}_{2}^{T} \dot{\tilde{f}}_{2} \\
= & \bar{z}_{1}^{T}\left(\dot{x}_{1}-\dot{x}_{1}^{f}-\dot{\xi}_{1}\right)+\bar{z}_{2}^{T}\left(\dot{x}_{2}-\dot{x}_{2}{ }^{f}-\dot{\xi}_{2}\right)+\frac{1}{c_{1}} \tilde{f}_{1}^{T} \dot{\tilde{f}}_{1}+\frac{1}{c_{2}} \tilde{f}_{2}^{T} \dot{\tilde{f}}_{2} \\
= & \bar{z}_{1}^{T}\left(f_{1}+g_{1} x_{2}+h_{1} u^{f}-\dot{x}_{1}{ }^{f}-\dot{\xi}_{1}\right)+\bar{z}_{2}^{T}\left(f_{2}+g_{2} u^{f}-\dot{x}_{2}{ }^{f}-\dot{\xi}_{2}\right)+\frac{1}{c_{1}} \tilde{f}_{1}^{T} \dot{\tilde{f}}_{1}+\frac{1}{c_{2}} \tilde{f}_{2}^{T} \dot{\tilde{f}}_{2} \\
= & \bar{z}_{1}^{T}\left(-\tilde{f}_{1}+\hat{f}_{1}+g_{1} z_{2}+g_{1} x_{2}{ }^{f}+h_{1} u^{f}-\dot{x}_{1}^{f}-\dot{\xi}_{1}\right)+\bar{z}_{2}{ }^{T}\left(-\tilde{f}_{2}+\hat{f}_{2}+g_{2} u^{f}-\dot{x}_{2}{ }^{f}-\dot{\xi}_{2}\right)+\frac{1}{c_{1}} \tilde{f}_{1}^{T} \dot{f}_{1}+\frac{1}{c_{2}} \tilde{f}_{2}^{T} \dot{\tilde{f}}_{2} \\
= & \bar{z}_{1}^{T}\left(-\tilde{f}_{1}+\hat{f}_{1}+g_{1} z_{2}+g_{1} x_{2}{ }^{f}-g_{1} x_{2}{ }^{\bmod }+g_{1} x_{2}{ }^{d}-g_{1} \xi_{2}+h_{1} u^{f}-\dot{x}_{1}{ }^{f}-\dot{\xi}_{1}\right) \\
& +\bar{z}_{2}^{T}\left(-\tilde{f}_{2}+\hat{f}_{2}+g_{2} u^{f}-g_{2} u^{f}+g_{2} u^{d}-\dot{x}_{2}{ }^{f}-\dot{\xi}_{2}\right)+\frac{1}{c_{1}} \tilde{f}_{1}^{T} \dot{\tilde{f}}_{1}+\frac{1}{c_{2}} \tilde{f}_{2}^{T} \dot{\tilde{f}}_{2}
\end{aligned}
$$


It is to be noted that the filtered state $x_{2}^{f}$ and $u^{f}$ are different from the desired states $x_{2}^{d}$ and $u^{d}$ in contrast to the unconstrained adaptive backstepping controller. By applying Eqs. (41)-(46) to Eq. (48) yields,

$$
\begin{aligned}
\dot{V}_{3}= & \bar{z}_{1}^{T}\left\{-\tilde{f}_{1}+g_{1} z_{2}+g_{1}\left(x_{2}{ }^{f}-x_{2}{ }^{\text {mod }}\right)-g_{1} \xi_{2}-K_{1} z_{1}-\dot{\xi}_{1}\right\} \\
& +\bar{z}_{2}^{T}\left\{-\tilde{f}_{2}+g_{2}\left(u^{f}-u^{d}\right)-g_{1} \bar{z}_{1}-K_{2} z_{2}-\dot{\xi}_{2}\right\}+\tilde{f}_{1}^{T} \bar{z}_{1}+\tilde{f}_{2}^{T} \bar{z}_{2} \\
= & \bar{z}_{1}^{T}\left\{g_{1}\left(z_{2}-\xi_{2}\right)-K_{1}\left(z_{1}-\xi_{1}\right)\right\}+\bar{z}_{2}^{T}\left\{g_{1}^{T} \bar{z}_{1}-K_{2}\left(z_{2}-\xi_{2}\right)\right\} \\
= & -\bar{z}_{1}^{T} K_{1} \bar{z}_{1}-\bar{z}_{2}^{T} K_{2} \bar{z}_{2} \leq 0
\end{aligned}
$$

Finally, the time derivative of Eq. (47) is negative semidefinite. The state errors defined in Eqs. (38) and (39) are regulated. Therefore $x_{1}$ converges to $x_{1}^{d}$, and $x_{2}$ converges to $x_{2}^{d}$, respectively. The detailed procedure of deriving Eqs. (48) and (49) is given in Appendix.

Figure 3 illustrates the schematic diagram of the constrained adaptive backstepping controller from the reference command $x_{1}^{d}$ to the filtered control input $u^{f}$. When the desired states and the filtered states are same, the desired control input $u^{d}$ in Eq. (44) makes $x_{2}$ to converge to $x_{2}^{d}$ in Eq. (43). When the desired states and filtered states are different, $\xi_{1}$ and $\xi_{2}$ in Eqs. (40)-(42) are activated. Adaptation errors in Eqs. (38) and (39) are compensated until the desired states in Eqs. (43) and (44) become feasible states. Consequently, the compensating variables $\xi_{1}$ and $\xi_{2}$ prevent the estimation errors from aggressive adaptation.

\section{Simulation Result and Analysis}

In order to verify the performance of the proposed control law, six degree-of-freedom nonlinear simulations are performed. The proposed constrained adaptive backstepping controller is compared with the gain-scheduled classical controller, the non-adaptive backstepping controller, and the unconstrained adaptive backstepping controller. The gainscheduled controller is designed by using Proportional-and-
Integral (PI) controller [26]. The non-adaptive backstepping controller is designed by assuming the estimation errors in Eqs. (21)-(22) zero and also by replacing $f_{1}$ and $f_{2}$ in Eqs. (25) and (31) with $f_{1}$ and $f_{2}$, respectively. The unconstrained and constrained adaptive backstepping controllers are designed as described in Sections 3.1 and 3.2, respectively.

Windgustandactuatorfaultareconsideredinthesimulation of the NASA HL-20 model [25]. The NASA HL-20 simulation model contains WGS84 gravity model, COESA(Committee on Extension to the Standard Atmosphere) atmosphere model, and various wind models that include the wind shear model, the Dryden wind turbulence model, and the discrete wind gust model $[25,26]$. The wind shear model is implemented for the Category $\mathrm{C}$ terminal flight phase according to the Military Specification MIL-F-8785C. The magnitude of the shear wind is defined as follows,

$$
V_{\text {shear }}=W_{0} \frac{\ln \left(h / z_{0}\right)}{\ln \left(20 / z_{0}\right)}
$$

where, $V_{\text {shear }}$ is the magnitude of the shear wind, $W_{0}$ is a measured wind at the altitude of $20 \mathrm{ft}, h$ is the altitude of the aircraft, and $z_{0}$ is 0.15 for Category $\mathrm{C}$ terminal flight phase. The Dryden wind turbulence and gust are generated by using the velocity spectra and transfer function according to the MIL-F-8785C. The gust is defined as follows,

$$
V_{\text {gust }}=\left\{\begin{array}{cl}
0 & , x_{g}<0 \\
\frac{V_{m}}{2}\left[1-\cos \left(\frac{\pi x_{g}}{d_{m}}\right)\right] & , 0 \leq x_{g} \leq d_{m} \\
V_{m} & , x_{g}>d_{m}
\end{array}\right.
$$

where, $V_{\text {gust }}$ is the magnitude of the gust wind, $V_{m}$ is the gust amplitude, $d_{m}$ is the gust length, and $x_{g}$ is the traveled distance. In this simulation, the gust length is determined as $120 \mathrm{~m}, 120 \mathrm{~m}$, and $80 \mathrm{~m}$ in the $X, Y$, and $Z$ directions of the body frame, respectively. The gust amplitude is varied from zero to $55 \mathrm{~m} / \mathrm{s}$.

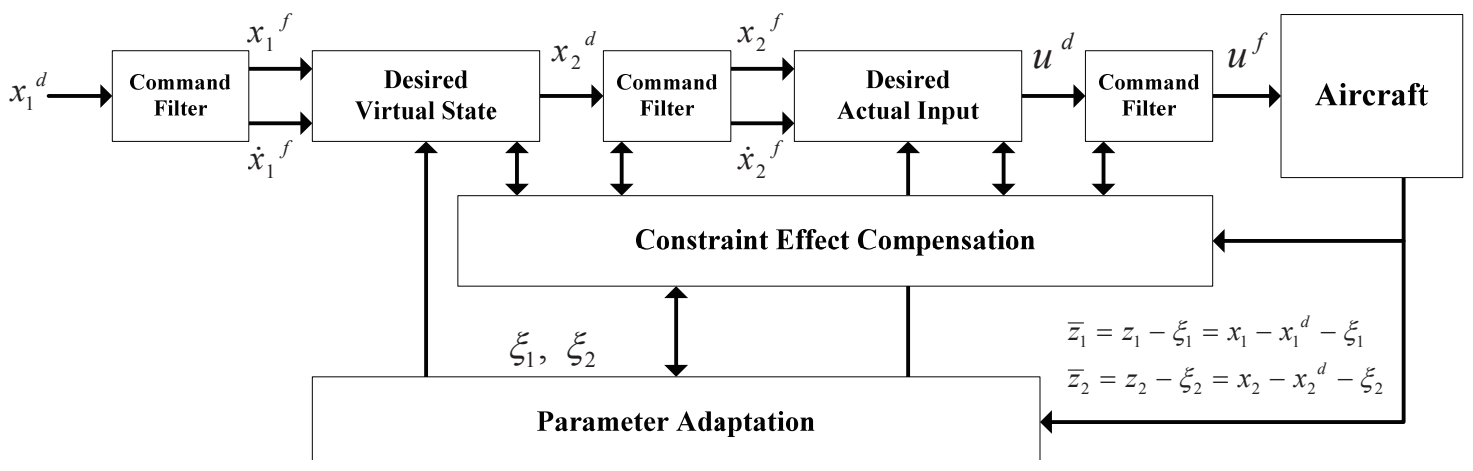

Fig. 3. Constrained adaptive backstepping control scheme 


\subsection{Simulation I: Wind Disturbance Case}

The landing performance of each controller is compared to the case when the aircraft encounters an unknown wind gust. The glide slope is defined as a function of the range from the current position of the aircraft to the desired touchdown point [26]. The desired attitude of the aircraft along the glide slope is considered as follows,

$$
x_{1}^{d}=\left[\begin{array}{lll}
\phi_{d} & \alpha_{d} & \beta_{d}
\end{array}\right]^{T}
$$

where, $\emptyset_{d}$ is a desired roll angle, $\alpha_{d}$ is the desired angle of attack, and $\beta_{d}$ is the desired sideslip angle. The desired roll angle is generated for the regulation of the cross-range from the aircraft position to the runway center. The initial $\emptyset_{d}$ is zero because the aircraft is assumed to be aligned to the center of the runway at the start of the landing. $\beta_{d}$ is always kept to be zero. The initial condition of the aircraft is chosen as,

$$
\left[\begin{array}{lll}
p_{x} & p_{y} & p_{z}
\end{array}\right]=\left[\begin{array}{lll}
12,072 & 0 & 3,048
\end{array}\right] \text { (meter) }
$$
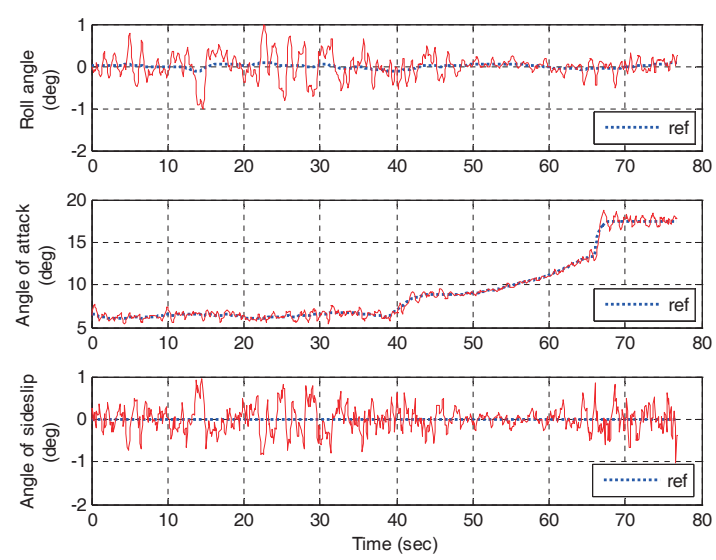

Fig. 4. Command and response: gain-scheduled PI controller (no gust)
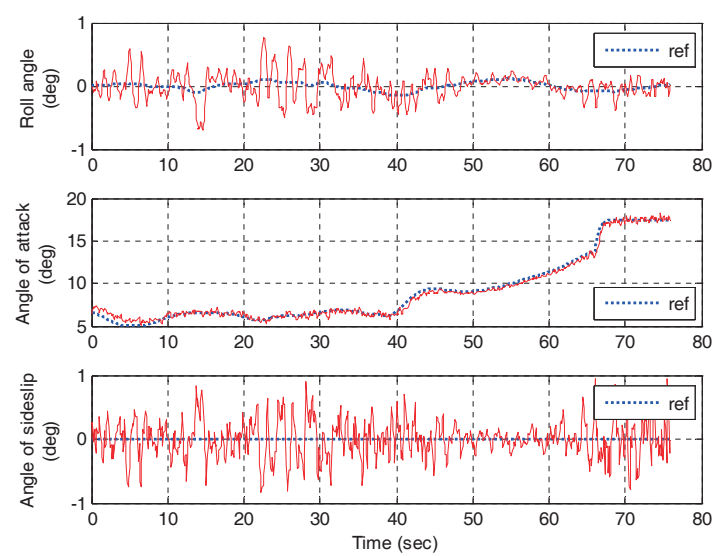

Fig. 5. Command and response: constrained adaptive backstepping controller (no gust)

$$
\begin{aligned}
& {\left[\begin{array}{lll}
u_{0} & v_{0} & w_{0}
\end{array}\right]=\left[\begin{array}{lll}
202.67 & 0.0 & 23.257
\end{array}\right](\mathrm{m} / \mathrm{s})} \\
& {\left[\begin{array}{lll}
\phi_{0} & \theta_{0} & \psi_{0}
\end{array}\right]=\left[\begin{array}{lll}
0 & -11.4 & 0
\end{array}\right](\mathrm{deg})}
\end{aligned}
$$

where, $\left(p_{x} p_{y} p_{z}\right)$ is the initial position of the aircraft, $\left(u_{0}\right.$ $v_{0} w_{0}$ ) is the initial velocity of the aircraft, and $\emptyset_{0}, \theta_{0}$, and $\psi_{0}$ are the initial roll angle, pitch angle, and yaw angle of the aircraft, respectively. All the initial angular velocities of the aircraft are set to be zero.

First, the wind turbulence is only considered and the wind gust is excluded. The landing performances of all the controllers are similar and they are as shown in Figs. 4-7. The control commands and the corresponding responses of the gain-scheduled PI controller and the constrained adaptive backstepping controller are shown in Figs. 4 and 5, respectively. The altitude, flight path angle, and the velocity of the aircraft are shown in Figs. 6 and 7, respectively.

When the wind gust with a magnitude of $55 \mathrm{~m} / \mathrm{s}$ is included in the simulation for 22 seconds, the tracking error
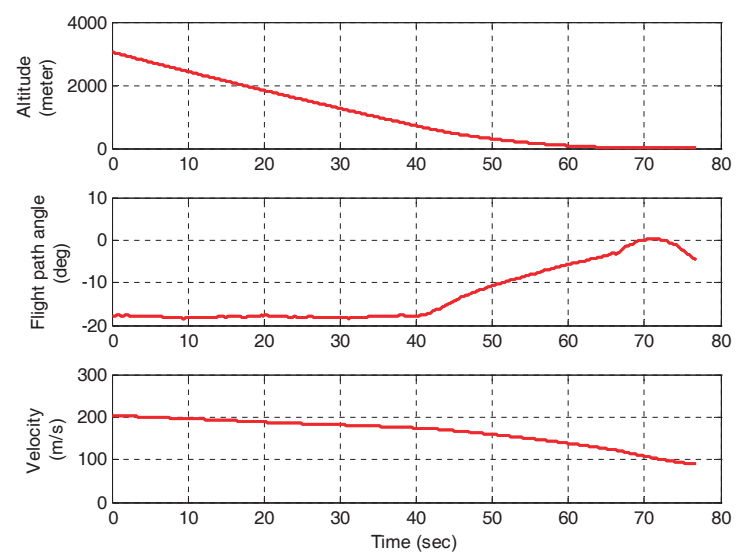

Fig. 6. Altitude, flight path angle, and velocity: gain-scheduled PI controller (no gust)
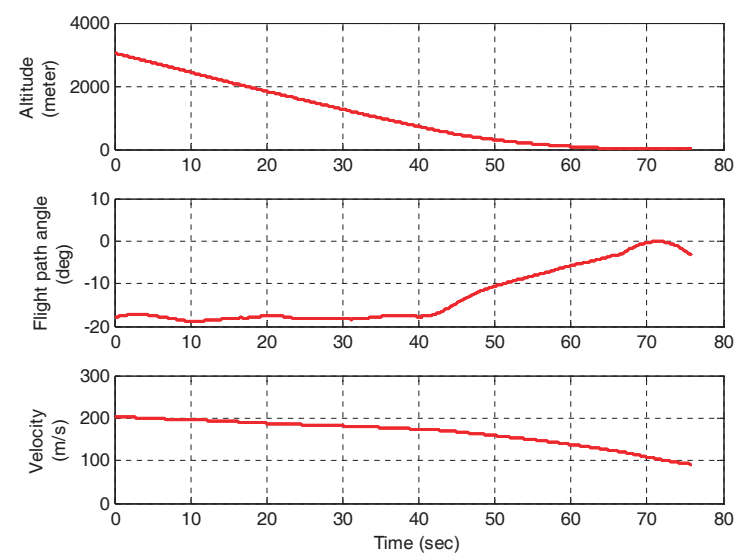

Fig. 7. Altitude, flight path angle, and velocity: constrained adaptive backstepping controller (no gust) 
of the gain-scheduled PI controller increases. It is greater compared to that of the three backstepping controllers. The aircraft states of the gain-scheduled PI controller diverge at the final landing phase are as shown in Fig. 8. On the other hand, all the three backstepping controllers overcome the wind gust and they land successfully. This is as shown in Fig. 9. As shown in Fig. 8, the states diverge due to the pitch oscillation which cannot be stabilized in the gain-scheduled PI controller. On the other hand, the states do not diverge as a result of the parameter adaptation and constraint compensation in the constrained adaptive backstepping controller which is as shown in Fig. 9.

In order to compare the landing performance against the wind gust, a tracking performance index is defined as the following square sum of the tracking errors

$$
J=\frac{1}{t_{f}} \int_{0}^{t_{f}}\left\{\sqrt{\left(\phi-\phi_{d}\right)^{2}}+\sqrt{\left(\alpha-\alpha_{d}\right)^{2}}+\sqrt{\left(\beta-\beta_{d}\right)^{2}}\right\} d t
$$

where, $t_{f}$ is the total simulation time. The performance indices of four controllers are summarized in Table 3. As shown in Table 3, when the magnitude of wind gust is smaller than $16.5 \mathrm{~m} / \mathrm{s}$, the landing performances of the
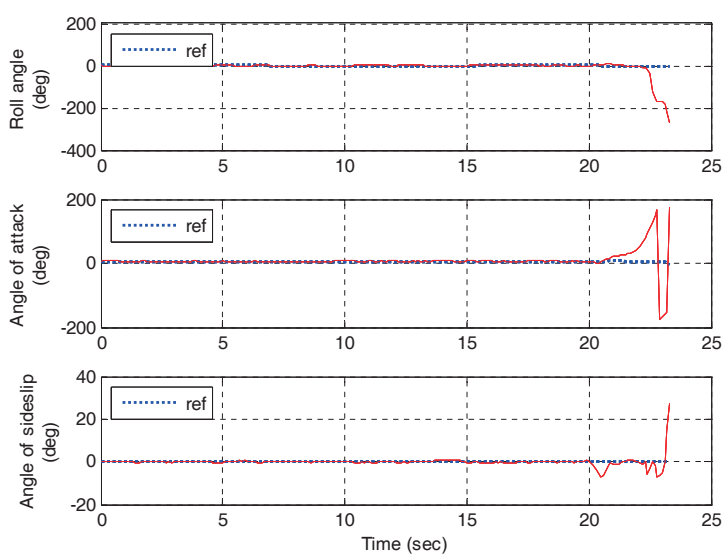

Fig. 8. Command and response: gain-scheduled PI controller (gust velocity $=55 \mathrm{~m} / \mathrm{s}$ ) four controllers are similar. However, for the wind gust of magnitude greater than $16.5 \mathrm{~m} / \mathrm{s}$, the tracking error of the gain-scheduled PI controller increases steeply compared to that of the three backstepping controllers. The tracking error of the unconstrained adaptive backstepping controller is less than that of the non-adaptive backstepping controller when the magnitude of wind gust is smaller than $16.5 \mathrm{~m} / \mathrm{s}$ because the small estimation errors in Eqs. (21) and (22) are well adapted. On the other hand, for the wind gust of magnitude greater than $16.5 \mathrm{~m} / \mathrm{s}$, the tracking error of the unconstrained adaptive backstepping controller is greater than that of the non-adaptive backstepping controller because of the large estimation error. This error occurs due to the wind gust which causes an overreacting adaptation. The non-adaptive backstepping controller provides an excellent tracking performance as long as the nonlinear functions $f_{1}, f_{2}, g_{1}, g_{2}$, $h_{1}$ in Eqs. (14) and (15) are known and this is assumed in this particular simulation. It is to be noted that the tracking error of the constrained adaptive backstepping is the smallest in most of the cases. This is due to the parameter adaptation and the constraint compensation which is described in Eqs. (38)-(46).
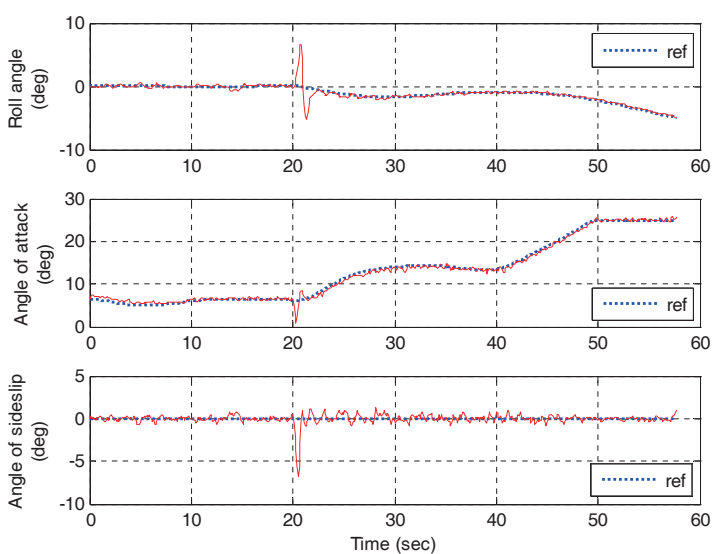

Fig. 9. Command and response: constrained adaptive backstepping controller (gust velocity $=55 \mathrm{~m} / \mathrm{s}$ )

Table 3. Comparison of the performance index according to the magnitude of wind gust

\begin{tabular}{|c|c|c|c|c|}
\hline $\begin{array}{c}\text { Magnitude of Wind Gust } \\
(\mathrm{m} / \mathrm{s})\end{array}$ & $\begin{array}{c}\text { Gain-scheduled } \\
\text { PI Controller }\end{array}$ & $\begin{array}{c}\text { Non-adaptive } \\
\text { Backstepping } \\
\text { Controller }\end{array}$ & $\begin{array}{c}\text { Unconstrained } \\
\text { Adaptive Backstepping } \\
\text { Controller }\end{array}$ & $\begin{array}{c}\text { Constrained Adaptive } \\
\text { Backstepping } \\
\text { Controller }\end{array}$ \\
\hline 0 & 3.0082 & 3.1576 & 2.9356 & 2.9359 \\
\hline 5.5 & 2.8374 & 3.0564 & 2.9647 & 2.5919 \\
\hline 16.5 & 3.4136 & 3.3671 & 3.4253 & 3.0114 \\
\hline 22.0 & 4.9744 & 3.9171 & 4.0764 & 3.5970 \\
\hline 27.5 & 14.1618 & 4.8141 & 5.1072 & 4.5014 \\
\hline 33.0 & 32.9586 & 5.7361 & 5.8489 & 5.1446 \\
\hline 55.0 & $\infty$ & 10.0467 & 12.4141 & 11.6010 \\
\hline
\end{tabular}



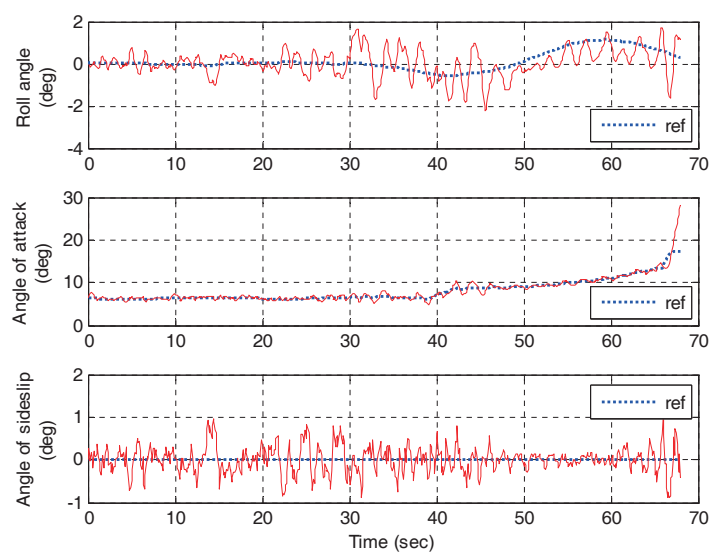

Fig. 10. Command and response: gain-scheduled PI controller (stuck angle $=10^{\circ}$ )
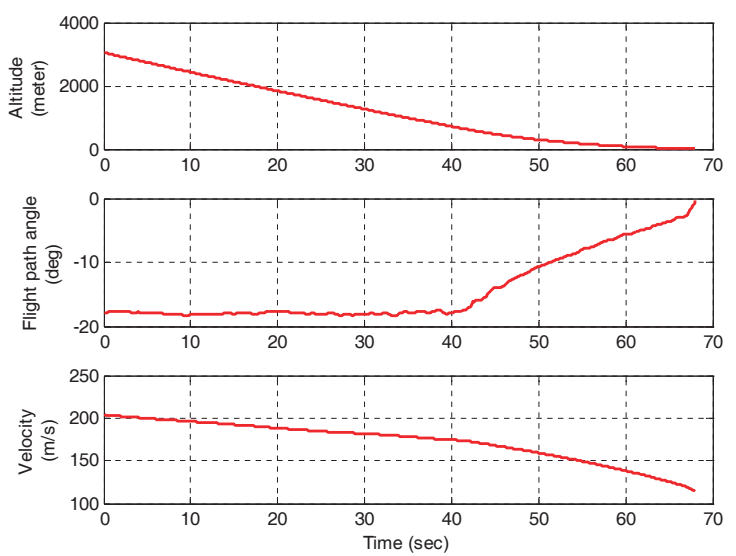

Fig. 11. Altitude, flight path angle, and velocity: gain-scheduled PI controller (stuck angle $=10^{\circ}$ )
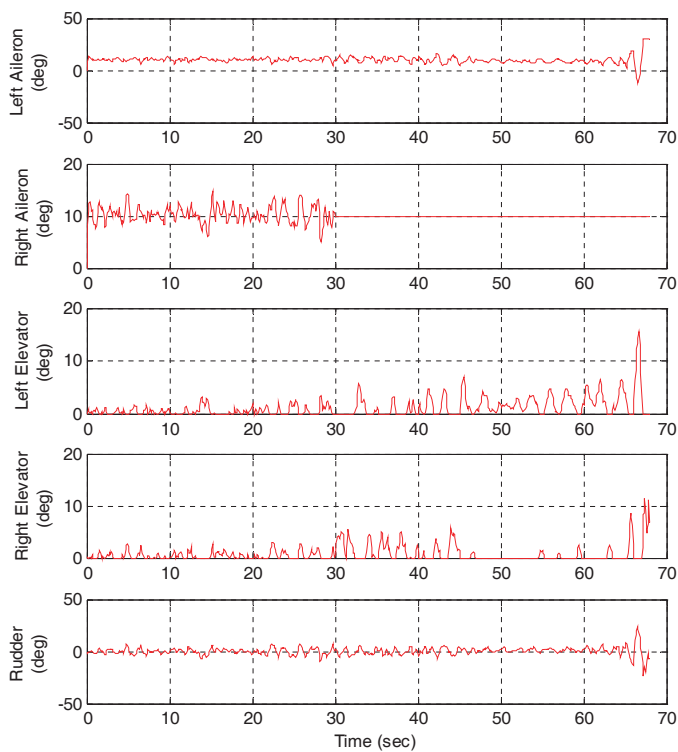

Fig. 12. Control surfaces: gain-scheduled PI controller (stuck angle $=$ $\left.10^{\circ}\right)$
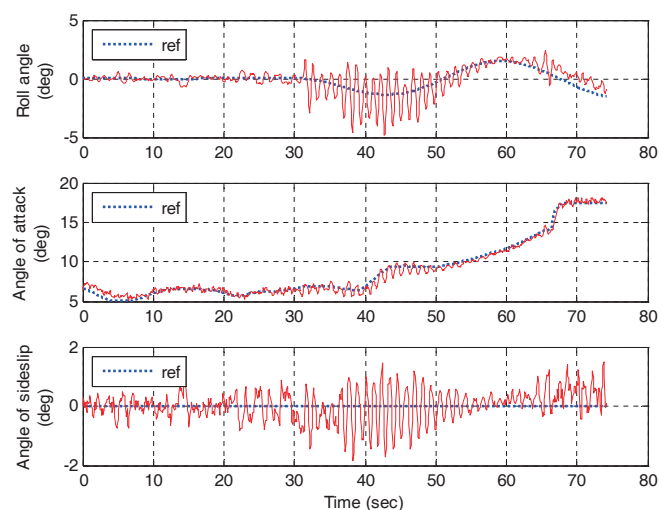

Fig. 13. Command and response: constrained adaptive backstepping controller (stuck angle $=10^{\circ}$ )
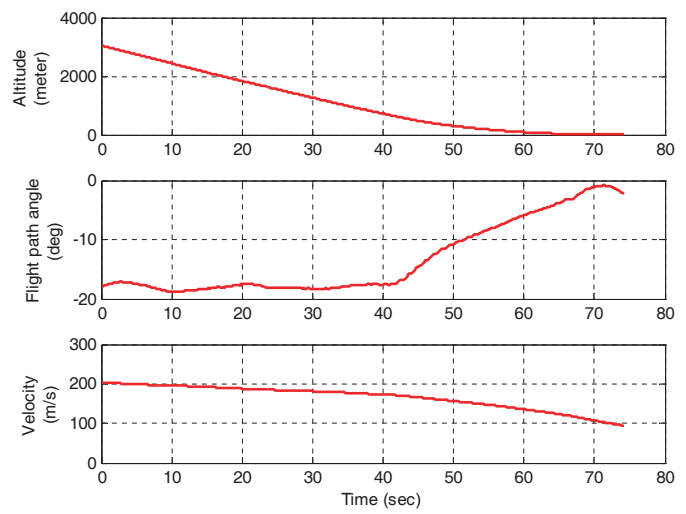

Fig. 14. Altitude, flight path angle, and velocity: constrained adaptive backstepping controller (stuck angle $=10^{\circ}$ )
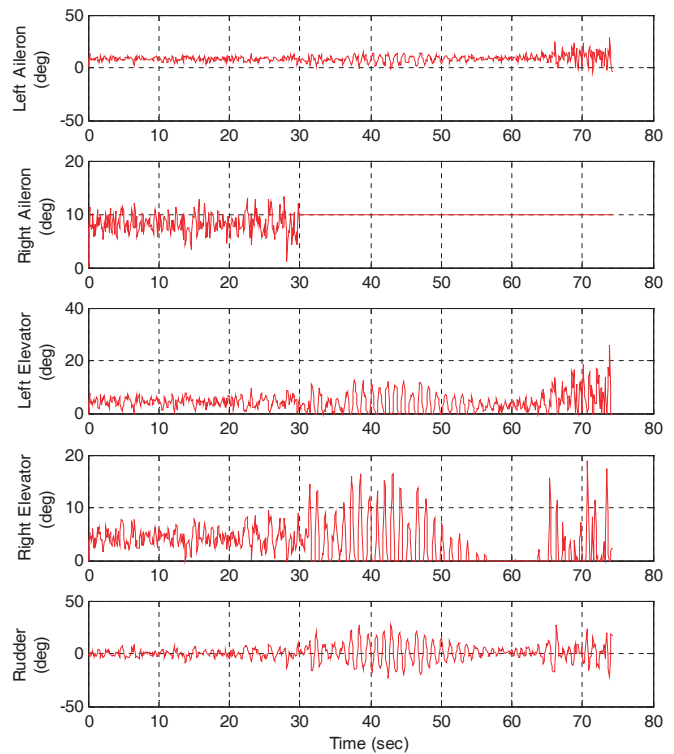

Fig. 15. Control surfaces: constrained adaptive backstepping controller (stuck angle $=10^{\circ}$ ) 

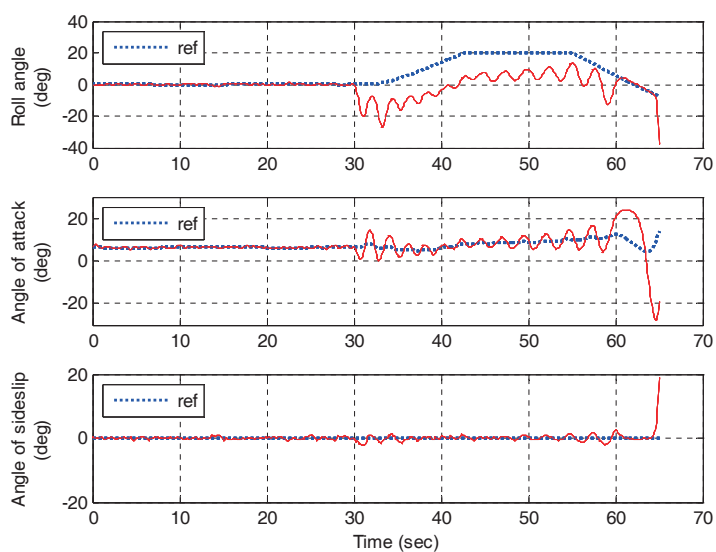

Fig. 16. Command and response: gain-scheduled PI controller (stuck angle $=30^{\circ}$ )
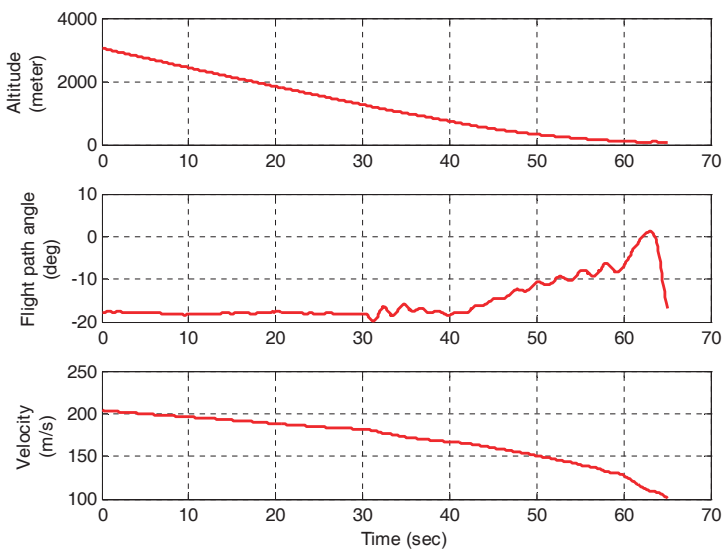

Fig. 17. Altitude, flight path angle, and velocity: gain-scheduled PI controller (stuck angle $=30^{\circ}$ )
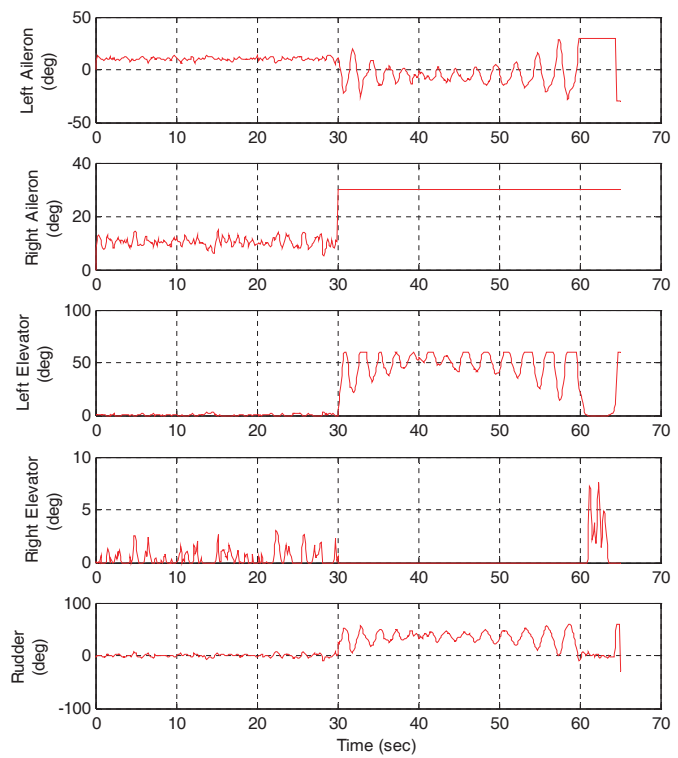

Fig. 18. Control surfaces: gain-scheduled PI controller (stuck angle = $\left.30^{\circ}\right)$

\subsection{Simulation II: Actuator Stuck Case}

The landing performance of each controller is compared to the case where the aircraft encounters with an actuator fault. In this simulation, the actuator fault of the right aileron occurs during the landing phase. The right aileron is assumed to be stuck at a certain position. In this study, the fault detection and isolation logic of the control surface is not considered. Instead, the fault-tolerant performance of the proposed control law is evaluated to overcome the actuator stuck. In this simulation, the deflection angle of both the right and left ailerons is positive in the direction of trailing-edgedown, the deflection angle of the both right and left elevators is positive in the direction of trailing-edge-down, and the deflection angle of the rudder is positive in the direction of trailing-edge-left, respectively.

When the right aileron operating around $10^{\circ}$, is stuck at the position of $10^{\circ}$ at 30 seconds, the landing performances of the gain-scheduled PI controller, the non-adaptive backstepping controller and the constrained adaptive backstepping controller are similar. The control responses, states, and control surfaces of the gain-scheduled PI controller are shown in Figs. 10, 11, and 12, respectively. Control responses, states, and control surfaces of the constrained adaptive backstepping controller are shown in Figs. 13, 14, and 15, respectively. As shown in Figs. 12 and 15, the angle of the right aileron is constant $10^{\circ}$ after 30 seconds and this is due to the actuator stuck. Even though the right aileron is stuck, the aircraft that uses both the controllers tracks the glide slope successfully and it is as shown in Figs. 10 and 13.

As the failure angle of the right aileron increases, the tracking error of the gain-scheduled PI controller becomes larger compared to the tracking errors of the constrained adaptive backstepping controller. When the right aileron is stuck at the position of $30^{\circ}$ at 30 seconds, the aircraft landing of the gain-scheduled PI controller cannot be performed while the landings of the three backstepping controllers are successfully performed. The control responses, states, and control surfaces of the gain-scheduled PI controller when the stuck angle is $30^{\circ}$ are shown in Figs. 16, 17, and 18 , respectively. The control responses, states, and control surfaces of the constrained adaptive backstepping controller are shown in Figs. 19, 20, and 21, respectively. As shown in Figs. 18 and 21, the angle of the right aileron after 30 seconds is $30^{\circ}$ and this is due to the actuator fault. As shown in Fig. 16, the aircraft of the gain-scheduled PI controller cannot track the roll angle command. As a result this tracking error causes a large error and an oscillation in tracking the angle-of-attack command. On the other hand, the aircraft of the constrained adaptive backstepping controller tracks the roll angle as well 
as the angle-of-attack commands. Finally, even though the right aileron is stuck it lands successfully on the runway.

In order to compare the landing performance against the actuator fault, the performance indices of the four controllers
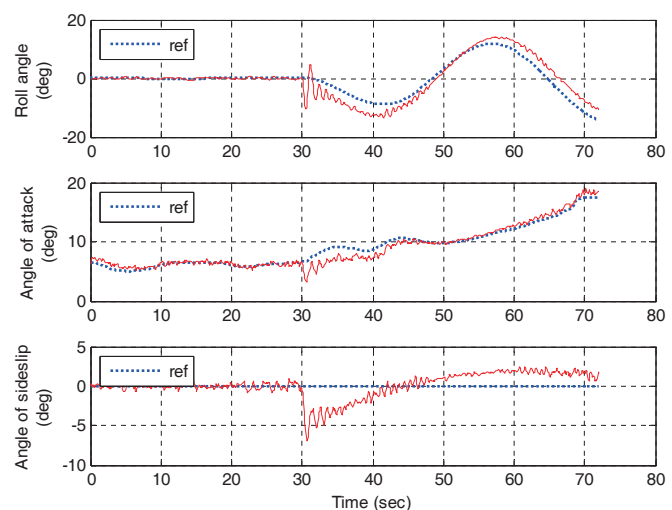

Fig. 19. Command and response: constrained adaptive backstepping controller (stuck angle $=30^{\circ}$ )
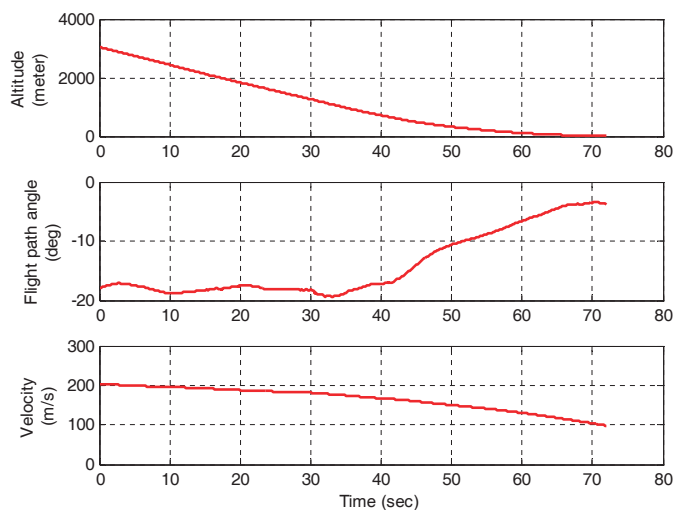

Fig. 20. Altitude, flight path angle, and velocity: constrained adaptive backstepping controller (stuck angle $=30^{\circ}$ ) are summarized in Table 4. In all the cases, the right aileron was operating around $10^{\circ}$ before the occurrence of the actuator stuck. As shown in Table 4, the tracking error of the gain-scheduled PI controller increases steeply as the stuck angle increases. This is due to the actuator fault which is not considered in the gain design process. The tracking error of the non-adaptive backstepping controller is less compared to that of the gain-scheduled PI controller even though the adaptation is not considered. The tracking error of the unconstrained adaptive backstepping controller is greater than that of the gain-scheduled PI controller for the stuck angle of 0,5 , and $10 \mathrm{deg}$, and it is greater than the tracking error of the non-adaptive backstepping controller for all the
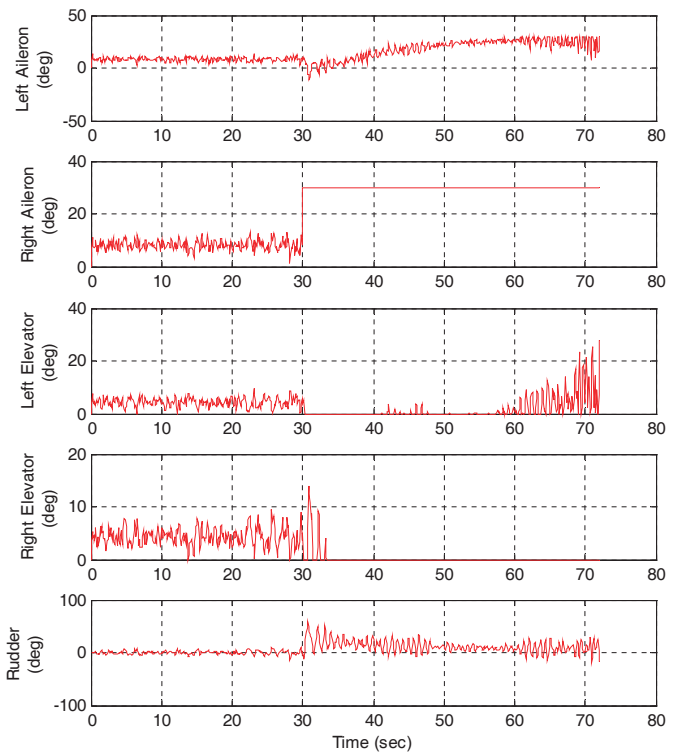

Fig. 21. Control surfaces: constrained adaptive backstepping controller (stuck angle $=30^{\circ}$ )

Table 4. Comparison of the performance index according to the stuck angle of the right aileron

\begin{tabular}{|c|c|c|c|c|}
\hline $\begin{array}{c}\text { Stuck Angle of Right } \\
\text { Aileron } \\
(\mathrm{deg})\end{array}$ & $\begin{array}{c}\text { Gain-scheduled } \\
\text { PI Controller }\end{array}$ & $\begin{array}{c}\text { Non-adaptive } \\
\text { Backstepping } \\
\text { Controller }\end{array}$ & $\begin{array}{c}\text { Unconstrained } \\
\text { Adaptive Backstepping } \\
\text { Controller }\end{array}$ & $\begin{array}{c}\text { Constrained Adaptive } \\
\text { Backstepping } \\
\text { Controller }\end{array}$ \\
\hline 0 & $\begin{array}{c}433.0091 \\
\left(t_{f}=68 \mathrm{~s}, P_{z, f}=16.3 \mathrm{~m}\right)\end{array}$ & 45.7147 & 625.2133 \\
\hline 5 & $\begin{array}{c}115.5080 \\
\left(t_{f}=68 \mathrm{~s}, P_{z, f}=14.0 \mathrm{~m}\right)\end{array}$ & 16.1483 & 245.3531 & 13.9505 \\
\hline 10 & $\begin{array}{c}14.2794 \\
\left(t_{f}=68 \mathrm{~s}, P_{z, f}=15.1 \mathrm{~m}\right)\end{array}$ & 13.4912 & 160.7171 \\
\hline $\begin{array}{c}88.1755 \\
\left(t_{f}=68 \mathrm{~s}, P_{z, f}=14.4 \mathrm{~m}\right)\end{array}$ & 32.6069 & 53.7391 & 22.4694 \\
\hline 20 & $\begin{array}{c}288.8677 \\
\left(t_{f}=68 \mathrm{~s}, P_{z, f}=14.3 \mathrm{~m}\right)\end{array}$ & 74.8482 & 68.2153 & 43.4900 \\
\hline 30 & $\begin{array}{c}1218.7 \\
\left(t_{f}=62 \mathrm{~s}, P_{z, f}=87.3 \mathrm{~m}\right)\end{array}$ & 211.0650 & 170.2643 & 97.3661 \\
\hline
\end{tabular}


cases. The poor performance of the unconstrained adaptive backstepping controller is due to the fact that the physical constraints of the control surfaces are not considered at all in the parameter adaptation. Unconstrained adaptation makes the system unstable and the situation worse. Finally, the tracking error of the constrained adaptive backstepping is the smallest for all the stuck angles and this is due to the parameter adaptation and the constraint compensation that is described in Eqs. (38)-(46).

\section{Conclusion}

Adaptive backstepping controller is designed to make the fixed-wing aircraft land on the runway in the presence of wind gust and actuator stuck. The nonlinear six degreeof-freedom dynamics of the aircraft is considered in the design of the backstepping controller. The adaptive scheme is applied to the backstepping controller in order to deal with the modeling errors in the aircraft dynamics and the external disturbances. In the parameter adaptation, the dynamic characteristics and the constraints of the aircraft states and actuator inputs are taken into account to prevent aggressive adaptation and provide a reliable landing. In order to verify the performance of the proposed control law numerical simulations are performed by using the nonlinear six degree-of-freedom aircraft model. The constrained adaptive backstepping controller successfully overcomes the wind gust and the actuator fault while the gain-scheduled PI controller, the non-adaptive backstepping controller, and the unconstrained adaptive backstepping controller cannot handle it.

\section{Acknowledgements}

This work was supported by the Korea Science and Engineering Foundation (KOSEF) grant funded by the Korea government (MEST) (No. R0A-2007-000-10017-0).

\section{References}

[1] Statistical Summary of Commercial Jet Airplane Accidents: Worldwide Operations 1959-2001, Airplane Safety Division, Boeing Commercial Airplane, Seattle, Washington,
June 2002, pp. 17-22, URL:http://www.boeing.com/aboutus/ govt_ops/reports_white_papers/commercial_jet_airplane_ accidents_statistical_summary.pdf [cited 27 March 2012].

[2] Stevens, B. L., and Lewis, F. L., Aircraft Control and Simulation, Second Edition, John Wiley \& Sons, Hoboken, NJ, 2003.

[3] Niewoehner, R. J., and Kaminer I., "Design of an Autoland Controller for Carrier-Based F-14 Aircraft Using $H_{\infty}$ Output-Feedback Synthesis", American Control Conference, Baltimore, MD, June 1994.

[4] Fialho, I., Balas, G. J., Packard, A. K., Renfrow, J., and Mullaney C., "Gain-Scheduled Lateral Control of the F-14 Aircraft During Powered Approach Landing", Journal of Guidance, Control, and Dynamics, Vol. 23, No. 3, 2000, pp. 450-458.

[5] Liao, F., Wang, J. L., Poh, E. K., and Li, D., "FaultTolerant Robust Automatic Landing Control Design", Journal of Guidance, Control, and Dynamics, Vol. 28, No. 5, 2005, pp. 854-871.

[6] Wagner, T., and Valasek, J., "Digital Autoland Control Laws Using Quantitative Feedback Theory and Direct Digital Design", Journal of Guidance, Control, and Dynamics, Vol. 30, No. 5, 2007, pp. 1399-1413.

[7] Khalil, H. K., Nonlinear Systems, Third Edition, Prentice Hall, Inc., Upper Saddle River, NJ, 2002.

[8] Krstic, M., Kanellakopoulos, I., and Kokotovic, P., Nonlinear and Adaptive Control Design, John Wiley \& Sons, Inc., New York, NY, 1995.

[9] Biju, B., and Pradeep, S., "Automatic Landing System Design using Feedback Linearization Method", AIAA Infotech@Aerospace 2007 Conference and Exhibit, Rohnert Park, CA, May 2007.

[10] Ju, H., Tsai, C., and Lee, C., "Flight Path Control Design for Glide-slope Tracking by Backstepping”, IEEE International Conference on Mechatronics, Taipei, Taiwan, July 2005.

[11] Saini, G., and Balakrishnan, S. N., "Adaptive Critic Based Neurocontroller for Autolanding of Aircrafts", American Control Conference, Albuquerque, NM, June 1997.

[12] Naikal, N. S., Panikkar, R., Pashilkar, A. A., and Nagaraj, R., "Improved Fault Tolerance for Autolanding Using Adaptive Backstepping Neural Controller", 16th IEEE International Conference on Control Applications, Singapore, October 2007.

[13] Yoon, S., Kim, Y., and Kim, S., "Pursuit Guidance Law and Adaptive Backstepping Controller Design for VisionBased Net-Recovery UAV", AIAA Guidance, Navigation, and Control Conference, Honolulu, HI, August 2008.

[14] Lee, D., Kim, H. J., and Sastry, S., "Feedback Linearization vs. Adaptive Sliding Mode Control for a Quadrotor Helicopter", International Journal of Control, 
Automation, and Systems, Vol. 7, No. 3, 2009, pp. 419-428.

[15] Nho, K., and Agarwal, R. K., "Automatic Landing System Design Using Fuzzy Logic", Journal of Guidance, Control, and Dynamics, Vol. 23, No. 2, 2000, pp. 298-304.

[16] Ha, C., “Gain-Scheduled Directional Guidance Controller Design Using a Genetic Algorithm for Automatic Precision Landing", International Journal of Control, Automation, and Systems, Vol. 8, No. 1, 2010, pp. 107-117.

[17] Johnson, E., and Calise, A. J., "Neural Network Adaptive Control of Systems with Input Saturation", American Control Conference, Arlington, VA, June 2001.

[18] Farrell, J., Sharma, M., and Polycarpou, M., "Backstepping-Based Flight Control with Adaptive Function Approximation", Journal of Guidance, Control, and Dynamics, Vol. 28, No. 6, 2005, pp. 1089-1102.

[19] Airplane Flying Handbook, "Chapter 8. Approaches and Landings", Federal Aviation Administration, FAAH-8083-3A, 2004, pp. 1-35, URL: http://www.faa. gov/library/manuals/aircraft/airplane_handbook/ media/faa-h-8083-3a-4of7.pdf [cited 27 March 2012].

[20] Lee, T., and Kim, Y., "Nonlinear Adaptive Flight Control Using Backstepping and Neural Networks Controller,"Journal of Guidance, Control, and Dynamics, Vol. 24, No. 4, 2001, pp. 675-682.

[21] Kdrason, S. P., and Annaswamy, A. M., "Adaptive Control in the Presence of Input Constraints", IEEE Transactions on Automatic Control, Vol. 39, No. 11, 1994, pp. 2325-2330.

[22] Annaswamy, A. M., and Wong, J. E., "Adaptive Control in the Presence of Saturation Nonlinearity", International Journal of Adaptive Control and Signal Processing, Vol. 11, No. 1, 1997, pp. 3-19.

[23] Polycarpou, M., Farrell, J., and Sharma, M., "On-Line Approximation Control of Uncertain Nonlinear Systems: Issues with Control Input Saturation", American Control Conference, Denver, CO, June 2003.

[24] Sonneveldt, L., Chu, Q. P., and Mulder, J. A., "Nonlinear Flight Control Design Using Constrained Adaptive Backstepping", Journal of Guidance, Control, and Dynamics, Vol. 30, No. 2, 2007, pp. 322-336.

[25] Jackson, E. B., and Cruz, C. L., "Preliminary Subsonic Aerodynamic Model for Simulation Studies of the HL-20 Lifting Body", NASA TM4302, Langley Research Center, Hampton, VA, August 1992.

[26] Gage, S., "NASA HL-20 Lifting Body Airframe Modeled with Simulink and the Aerospace Blockset", MATLAB Digest [online journal], Vol. 10, No. 4, 2002, URL: http://www. mathworks.com/company /newsletters/digest/july02/ [cited 27 March 2012].

\section{Appendix}

The design procedure of the constrained adaptive backstepping controller is explained below in detail. With the command filter defined in Eq. (35), the nonlinear equations of aircraft motion are,

$$
\begin{aligned}
& x_{2}=g_{1}^{-1}\left(-\hat{f}_{1}-h_{1} u^{f}+\dot{x}_{1}^{f}-K_{1} z_{1}\right) \\
& \dot{x}_{2}=f_{2}+g_{2} u^{f} .
\end{aligned}
$$

where, $u^{f}$ is the final control input to the aircraft and it is as shown in Fig. 3.

Lemma 1. For the system of Eq. (A.1) with the adaptation error $z_{1}$ defined in Eq. (36), $x_{1}$ converges to $x_{1}^{d}$ if the control input $u^{f}$ is known, and $x_{2}$ and $\dot{\tilde{f}}_{1}$ are defined as

$$
\begin{aligned}
& x_{2}=g_{1}^{-1}\left(-\hat{f}_{1}-h_{1} u^{f}+\dot{x}_{1}^{f}-K_{1} z_{1}\right) \\
& \dot{\tilde{f}}_{1}=c_{1} z_{1}
\end{aligned}
$$

where, $K_{1}$ is a positive definite gain matrix and $c_{1}$ is a positive constant.

Proof) Consider the following Lyapunov function candidate

$$
V_{4}=\frac{1}{2} z_{1}^{T} z_{1}+\frac{1}{2 c_{1}} \tilde{f}_{1}^{T} \tilde{f}_{1}
$$

Substituting Eqs. (21), (36) and (A.1) into the time derivative of Eq. (A.5) yields

$$
\begin{aligned}
\dot{V}_{4} & =z_{1}^{T} \dot{z}_{1}+\frac{1}{c_{1}} \tilde{f}_{1}^{T} \dot{\tilde{f}}_{1} \\
& =z_{1}^{T}\left(\dot{x}_{1}-\dot{x}_{1}^{f}\right)+\frac{1}{c_{1}} \tilde{f}_{1}^{T} \dot{\tilde{f}}_{1} \\
& =z_{1}^{T}\left(f_{1}+g_{1} x_{2}+h_{1} u^{f}-\dot{x}_{1}^{f}\right)+\frac{1}{c_{1}} \tilde{f}_{1}^{T} \dot{\tilde{f}}_{1} \\
& =z_{1}^{T}\left(-\tilde{f}_{1}+\hat{f}_{1}+g_{1} x_{2}+h_{1} u^{f}-\dot{x}_{1}^{f}\right)+\frac{1}{c_{1}} \tilde{f}_{1}^{T} \dot{\tilde{f}}_{1}
\end{aligned}
$$

Applying Eqs. (A.3) and (A.4) to Eq. (A.6) yields

$$
\begin{aligned}
\dot{V}_{4} & =z_{1}^{T}\left(-\tilde{f}_{1}-K_{1} z_{1}\right)+\tilde{f}_{1}^{T} z_{1} \\
& =-z_{1}^{T} K_{1} z_{1} \leq 0
\end{aligned}
$$

Lemma 2. For the system of Eqs. (A.1) and (A.2) that the filtered state does not exceed the desired state $\left(x_{2}^{f}=x_{2}^{d}, u^{f}=\right.$ $\left.u^{d}\right)$, if $x_{2}^{d}, u^{d}$, $\dot{\tilde{f}}_{1}$, and $\dot{\tilde{f}}_{2}$ are defined as

$$
x_{2}{ }^{d}=g_{1}^{-1}\left(-\hat{f}_{1}-h_{1} u^{f}+\dot{x}_{1}^{f}-K_{1} z_{1}\right)
$$




$$
\begin{aligned}
& u^{d}=g_{2}{ }^{-1}\left(-\hat{f}_{2}-g_{1}^{T} z_{1}-K_{2} z_{2}+\dot{x}_{2}^{f}\right) \\
& \dot{\tilde{f}}_{1}=c_{1} z_{1} \\
& \dot{\tilde{f}}_{2}=c_{2} z_{2}
\end{aligned}
$$

where, $K_{1}$ and $K_{2}$ are positive definite gain matrices, and $c_{1}$ and $c_{2}$ are positive constants, then $x_{1}$ converges to $x_{1}^{d}$, and $x_{2}$ converges to $x_{2}^{d}$, respectively.

Proof) Consider the following Lyapunov function candidate

$$
V_{5}=\frac{1}{2} z_{1}{ }^{T} z_{1}+\frac{1}{2} z_{2}{ }^{T} z_{2}+\frac{1}{2 c_{1}} \tilde{f}_{1}^{T} \tilde{f}_{1}+\frac{1}{2 c_{2}} \tilde{f}_{2}^{T} \tilde{f}_{2}
$$

Substituting Eqs. (21), (22), (36), (37), (A.1), and (A.2) into the time derivative of Eq. (A.12) yields

$$
\begin{aligned}
\dot{V}_{s} & =z_{1}^{T} \dot{z}_{1}+z_{2}^{T} \dot{z}_{2}+\frac{1}{c_{1}} \tilde{f}_{1}^{T} \dot{\tilde{f}}_{1}+\frac{1}{c_{2}} \tilde{f}_{2}^{T} \dot{\tilde{f}}_{2} \\
& =z_{1}^{T}\left(\dot{x}_{1}-\dot{x}_{1}^{f}\right)+z_{2}^{T}\left(\dot{x}_{2}-\dot{x}_{2}{ }^{f}\right)+\frac{1}{c_{1}} \tilde{f}_{1}^{T} \dot{\tilde{f}}_{1}+\frac{1}{c_{2}} \tilde{f}_{2}^{T} \dot{\tilde{f}}_{2} \\
& =z_{1}^{T}\left(f_{1}+g_{1} x_{2}+h_{1} u^{f}-\dot{x}_{1}^{f}\right)+z_{2}{ }^{T}\left(f_{2}+g_{2} u^{f}-\dot{x}_{2}{ }^{f}\right)+\frac{1}{c_{1}} \tilde{f}_{1}^{T} \dot{\tilde{f}}_{1}+\frac{1}{c_{2}} \tilde{f}_{2}^{T} \dot{\tilde{f}}_{2} \\
& =z_{1}^{T}\left(-\tilde{f}_{1}+\hat{f}_{1}+g_{1} z_{2}+g_{1} x_{2}{ }^{f}+h_{1} u^{f}-\dot{x}_{1}{ }^{f}\right)+z_{2}^{T}\left(-\tilde{f}_{2}+\hat{f}_{2}+g_{2} u^{f}-\dot{x}_{2}{ }^{f}\right)+\frac{1}{c_{1}} \tilde{f}_{1}^{T} \dot{\tilde{f}}_{1}+\frac{1}{c_{2}} \tilde{f}_{2}^{T} \dot{\tilde{f}}_{2} \\
& =z_{1}^{T}\left(-\tilde{f}_{1}+\hat{f}_{1}+g_{1} z_{2}+g_{1} x_{2}{ }^{d}+h_{1} u^{f}-\dot{x}_{1}{ }^{f}\right)+z_{2}^{T}\left(-\tilde{f}_{2}+\hat{f}_{2}+g_{2} u^{d}-\dot{x}_{2}{ }^{f}\right)+\frac{1}{c_{1}} \tilde{f}_{1}^{T} \dot{\tilde{f}}_{1}+\frac{1}{c_{2}} \tilde{f}_{2}^{T} \dot{\tilde{f}}_{2}
\end{aligned}
$$

\section{Applying Eqs. (A.8)-(A.11) to Eq. (A.13) yields}

$$
\begin{aligned}
\dot{V}_{5} & =z_{1}^{T}\left(-\tilde{f}_{1}+g_{1} z_{2}-K_{1} z_{1}\right)+z_{2}^{T}\left(-\tilde{f}_{2}-g_{1}^{T} z_{1}-K_{2} z_{2}\right)+\tilde{f}_{1}^{T} z_{1}+\tilde{f}_{2}^{T} z_{2} \\
& =-z_{1}^{T} K_{1} z_{1}-z_{2}{ }^{T} K_{2} z_{2} \leq 0
\end{aligned}
$$

Lemma 3. For the system of Eqs. (A.1) and (A.2) that the filtered control input does not exceed the desired control input $\left(u^{f}=u^{d}\right)$ and the filtered state exceeds the desired state $\left(x_{2}^{f} \neq x_{2}^{d}\right)$, if $\bar{z}_{1}, \xi_{1}, u^{d}$, and $\dot{\tilde{f}}_{1}$ are defined as

$$
\begin{aligned}
& \bar{z}_{1}=z_{1}-\xi_{1}=x_{1}-x_{1}^{f}-\xi_{1} \\
& \dot{\xi}_{1}=-K_{1} \xi_{1}+g_{1}\left(x_{2}^{f}-x_{2}^{d}\right) \\
& u^{d}=g_{2}^{-1}\left(-\hat{f}_{2}-g_{1}^{T} \bar{z}_{1}-K_{2} z_{2}+\dot{x}_{2}^{f}\right) \\
& \dot{\tilde{f}}_{1}=c_{1} \bar{z}_{1},
\end{aligned}
$$

Then, $\xi_{1}$ converges to zero, $x_{1}$ converges to $x_{1}^{d}$, and $x_{2}$ converges to $x_{2}^{d}$, respectively.

Proof) Consider the following Lyapunov function candidate

$$
V_{6}=\frac{1}{2} \bar{z}_{1}^{T} \bar{z}_{1}+\frac{1}{2} z_{2}^{T} z_{2}+\frac{1}{2 c_{1}} \tilde{f}_{1}^{T} \tilde{f}_{1}+\frac{1}{2 c_{2}} \tilde{f}_{2}^{T} \tilde{f}_{2}
$$

By substituting Eqs. (21), (22), (36), (37), (A.1), (A.2), and (A.15) into the time derivative of Eq. (A.19) yields,

$$
\begin{aligned}
\dot{V}_{6} & =\bar{z}_{1}^{T} \dot{\bar{z}}_{1}+z_{2}^{T} \dot{z}_{2}+\frac{1}{c_{1}} \tilde{f}_{1}^{T} \dot{\tilde{f}}_{1}+\frac{1}{c_{2}} \tilde{f}_{2}^{T} \dot{\hat{f}}_{2} \\
& =\bar{z}_{1}^{T}\left(\dot{x}_{1}-\dot{x}_{1}^{f}-\dot{\xi}_{1}\right)+z_{2}^{T}\left(\dot{x}_{2}-\dot{x}_{2}{ }^{f}\right)+\frac{1}{c_{1}} \tilde{f}_{1}^{T} \dot{\tilde{f}}_{1}+\frac{1}{c_{2}} \tilde{f}_{2}^{T} \dot{\tilde{f}}_{2} \\
& =\bar{z}_{1}^{T}\left(f_{1}+g_{1} x_{2}+h_{1} u^{f}-\dot{x}_{1}{ }^{f}-\dot{\xi}_{1}\right)+z_{2}^{T}\left(f_{2}+g_{2} u^{f}-\dot{x}_{2}{ }^{f}\right)+\frac{1}{c_{1}} \tilde{f}_{1}^{T} \dot{\tilde{f}}_{1}+\frac{1}{c_{2}} \tilde{f}_{2}^{T} \dot{\hat{f}}_{2} \\
& =\bar{z}_{1}^{T}\left(-\tilde{f}_{1}+\hat{f}_{1}+g_{1} z_{2}+g_{1} x_{2}{ }^{f}+h_{1} u^{f}-\dot{x}_{1}{ }^{f}-\dot{\xi}_{1}\right)+z_{2}^{T}\left(-\tilde{f}_{2}+\hat{f}_{2}+g_{2} u^{f}-\dot{x}_{2}{ }^{f}\right)+\frac{1}{c_{1}} \tilde{f}_{1}^{T} \dot{\hat{f}}_{1}+\frac{1}{c_{2}} \tilde{f}_{2}^{T} \dot{\hat{f}}_{2} \\
& =\bar{z}_{1}^{T}\left(-\tilde{f}_{1}+\hat{f}_{1}+g_{1} z_{2}+g_{1} x_{2}{ }^{f}-g_{1} x_{2}{ }^{d}+g_{1} x_{2}{ }^{d}+h_{1} u^{f}-\dot{x}_{1}^{f}-\dot{\xi}_{1}\right)+z_{2}^{T}\left(-\tilde{f}_{2}+\hat{f}_{2}+g_{2} u^{d}-\dot{x}_{2}{ }^{f}\right)+\frac{1}{c_{1}} \tilde{f}_{1}^{T} \dot{\tilde{f}}_{1}
\end{aligned}
$$

By applying Eqs. (A.8), (A.11), (A.16), (A.17) and (A.18) to Eq. (A.20) yields,

$$
\begin{aligned}
\dot{V}_{6} & =\bar{z}_{1}^{T}\left\{-\tilde{f}_{1}+g_{1} z_{2}+g_{1}\left(x_{2}{ }^{f}-x_{2}{ }^{d}\right)-K_{1} z_{1}-\dot{\xi}_{1}\right\}+z_{2}{ }^{T}\left(-\tilde{f}_{2}-g_{1}{ }^{T} \bar{z}_{1}-K_{2} z_{2}\right)+\tilde{f}_{1}^{T} \bar{z}_{1}+\tilde{f}_{2}^{T} z_{2} \\
& =\bar{z}_{1}^{T}\left\{g_{1} z_{2}+g_{1}\left(x_{2}{ }^{f}-x_{2}{ }^{d}\right)-K_{1} z_{1}+K_{1} \xi_{1}-g_{1}\left(x_{2}{ }^{f}-x_{2}{ }^{d}\right)\right\}+z_{2}{ }^{T}\left(-g_{1}{ }^{T} \bar{z}_{1}-K_{2} z_{2}\right) \\
& =\bar{z}_{1}^{T}\left\{g_{1} z_{2}-K_{1}\left(z_{1}-\xi_{1}\right)\right\}+z_{2}^{T}\left(-g_{1} \bar{z}_{1}-K_{2} z_{2}\right) \\
& =-\bar{z}_{1}^{T} K_{1} \bar{z}_{1}-z_{2}{ }^{T} K_{2} z_{2} \leq 0
\end{aligned}
$$

Note that Eq. (A.9) is replaced with Eq. (A.17), and Eq. (A.10) is replaced with Eq. (A.18).

Theorem 1. For the system of Eqs. (A.1) and (A.2) that the filtered state and control input exceed the desired values $\left(u^{f}\right.$ $\left.\neq u^{d}, x_{2}^{f} \neq x_{2}^{d}\right)$, if $\bar{z}_{2}, \xi_{1}, \xi_{2}, \mathrm{x}_{2}{ }^{\text {mod }}$, and $\dot{\tilde{f}}_{2}$ are defined as

$$
\begin{aligned}
& \bar{z}_{2}=z_{2}-\xi_{2}=x_{2}-x_{2}^{f}-\xi_{2} \\
& \dot{\xi}_{1}=-K_{1} \xi_{1}+g_{1}\left(x_{2}^{f}-x_{2}^{\mathrm{mod}}\right) \\
& \dot{\xi}_{2}=-K_{2} \xi_{2}+g_{2}\left(u^{f}-u^{d}\right) \\
& x_{2}^{\bmod }=x_{2}^{d}-\xi_{2} \\
& \dot{\tilde{f}}_{2}=c_{2} \bar{z}_{2},
\end{aligned}
$$

Then, $\xi_{1}$ and $\xi_{2}$ converge to zero, $x_{1}$ converges to $x_{1}^{d}$, and $x_{2}$ converges to $x_{2}^{d}$, respectively.

Proof) Consider the following Lyapunov function candidate

$$
V_{7}=\frac{1}{2} \bar{z}_{1}^{T} \bar{z}_{1}+\frac{1}{2} \bar{z}_{2}^{T} \bar{z}_{2}+\frac{1}{2 c_{1}} \tilde{f}_{1}^{T} \tilde{f}_{1}+\frac{1}{2 c_{2}} \tilde{f}_{2}^{T} \tilde{f}_{2}
$$

By substituting Eqs. (21), (22), (36), (37), (A.1), (A.2), (A.15), (A.22), and (A.25) into the time derivative of Eq. (A.27) yields, 


$$
\begin{aligned}
& \dot{V}_{7}=\bar{z}_{1}^{T} \dot{\bar{z}}_{1}+\bar{z}_{2}^{T} \dot{\bar{z}}_{2}+\frac{1}{c_{1}} \tilde{f}_{1}^{T} \dot{\tilde{f}}_{1}+\frac{1}{c_{2}} \tilde{f}_{2}^{T} \dot{\tilde{f}}_{2} \\
& =\bar{z}_{1}^{T}\left(\dot{x}_{1}-\dot{x}_{1}^{f}-\dot{\xi}_{1}\right)+\bar{z}_{2}^{T}\left(\dot{x}_{2}-\dot{x}_{2}^{f}-\dot{\xi}_{2}\right)+\frac{1}{c_{1}} \tilde{f}_{1}^{T} \dot{f}_{1}+\frac{1}{c_{2}} \tilde{f}_{2}^{T} \dot{\hat{f}}_{2} \\
& =\bar{z}_{1}^{T}\left(f_{1}+g_{1} x_{2}+h_{1} u^{f}-\dot{x}_{1}{ }^{f}-\dot{\xi}_{1}\right)+\bar{z}_{2}^{T}\left(f_{2}+g_{2} u^{f}-\dot{x}_{2}{ }^{f}-\dot{\xi}_{2}\right)+\frac{1}{c_{1}} \tilde{f}_{1}^{T} \dot{f}_{1}+\frac{1}{c_{2}} \tilde{f}_{2}^{T} \dot{f}_{2} \\
& =\bar{z}_{1}^{T}\left(-\tilde{f}_{1}+\hat{f}_{1}+g_{1} z_{2}+g_{1} x_{2}^{\prime}+h_{1} u^{\prime}-\dot{x}_{1}^{\prime}-\dot{\xi}_{1}\right)+\bar{z}_{2}^{T}\left(-\tilde{f}_{2}+\hat{f}_{2}+g_{2} u^{\prime}-\dot{x}_{2}{ }^{\prime}-\dot{\xi}_{2}\right)+\frac{1}{c_{1}} \tilde{f}_{1}^{T} \dot{\hat{f}}_{1}+\frac{1}{c_{2}} \tilde{f}_{2}^{T} \dot{\hat{f}}_{2} \\
& =\bar{z}_{1}^{T}\left(-\tilde{f}_{1}+\hat{f}_{1}+g_{1} z_{2}+g_{1} x_{2}^{f}-g_{1} x_{2}^{\text {mod }}+g_{1} x_{2}^{\text {mod }}+h_{1} u^{f}-\dot{x}_{1}^{f}-\dot{\xi}_{1}\right) \\
& +\bar{z}_{2}^{r}\left(-\tilde{f}_{2}+\hat{f}_{2}+g_{2} u^{\prime}-g_{2} u^{d}+g_{2} u^{d}-\dot{x}_{2}{ }^{\prime}-\dot{\xi}_{2}\right)+\frac{1}{c_{1}} \tilde{f}_{1}^{r} \dot{f}_{1}+\frac{1}{c_{2}} \tilde{f}_{2}^{r} \dot{\tilde{f}}_{2} \\
& =\bar{z}_{1}^{T}\left(-\tilde{f}_{1}+\hat{f}_{1}+g_{1} z_{2}+g_{1} x_{2}^{f}-g_{1} x_{2}{ }^{\text {mod }}+g_{1} x_{2}{ }^{d}-g_{1} \xi_{2}+h_{1} u^{f}-\dot{x}_{1}{ }^{\prime}-\dot{\xi}_{1}\right) \\
& +\bar{z}_{2}^{T}\left(-\tilde{f}_{2}+\hat{f}_{2}+g_{2} u^{f}-g_{2} u^{d}+g_{2} u^{d}-\dot{x}_{2}{ }^{f}-\dot{\xi}_{2}\right)+\frac{1}{c_{1}} \tilde{f}_{1}^{T} \dot{\tilde{f}}_{1}+\frac{1}{c_{2}} \tilde{f}_{2}^{T} \dot{\hat{f}}_{2}
\end{aligned}
$$

$$
\begin{aligned}
\dot{V}_{7}= & \bar{z}_{1}^{T}\left\{-\tilde{f}_{1}+g_{1} z_{2}+g_{1}\left(x_{2}{ }^{f}-x_{2}{ }^{\bmod }\right)-g_{1} \xi_{2}-K_{1} z_{1}-\dot{\xi}_{1}\right\} \\
& +\bar{z}_{2}^{T}\left\{-\tilde{f}_{2}+g_{2}\left(u^{f}-u^{d}\right)-g_{1}^{T} \bar{z}_{1}-K_{2} z_{2}-\dot{\xi}_{2}\right\}+\tilde{f}_{1}^{T} \bar{z}_{1}+\tilde{f}_{2}^{T} \bar{z}_{2} \\
= & \bar{z}_{1}^{T}\left\{g_{1} z_{2}+g_{1}\left(x_{2}{ }^{f}-x_{2}{ }^{\mathrm{mod}}\right)-g_{1} \xi_{2}-K_{1} z_{1}+K_{1} \xi_{1}-g_{1}\left(x_{2}{ }^{f}-x_{2}{ }^{\mathrm{mod}}\right)\right\} \\
& +\bar{z}_{2}^{T}\left\{g_{2}\left(u^{f}-u^{d}\right)-g_{1}^{T} \bar{z}_{1}-K_{2} z_{2}+K_{2} \xi_{2}-g_{2}\left(u^{f}-u^{d}\right)\right\} \\
= & \bar{z}_{1}^{T}\left\{g_{1}\left(z_{2}-\xi_{2}\right)-K_{1}\left(z_{1}-\xi_{1}\right)\right\}+\bar{z}_{2}^{T}\left\{g_{1}^{T} \bar{z}_{1}-K_{2}\left(z_{2}-\xi_{2}\right)\right\} \\
= & -\bar{z}_{1}^{T} K_{1} \bar{z}_{1}-\bar{z}_{2}^{T} K_{2} \bar{z}_{2} \leq 0
\end{aligned}
$$

Note that Eqs. (A.9), (A.10), (A.11), and (A.16) are replaced with Eq. (A.17), (A.18), (A.26), and (A.23), respectively.

By applying Eqs. (A.8), (A.17), (A.18), (A.23), (A.24) and (A.26) to Eq. (A.28) yields, 